\title{
Acinetobacter baumannii biofilms: effects of physicochemical factors, virulence, antibiotic resistance determinants, gene regulation, and future antimicrobial treatments
}

REVIEW

This article was published in the following Dove Press journal: Infection and Drug Resistance

\section{Emmanuel C Eze Hafizah Y Chenia ${ }^{2}$ Mohamed E El Zowalaty' \\ 'Virology and Microbiology Research Group, School of Health Sciences, College of Health Sciences, University of KwaZulu-Natal, Durban, South Africa; ${ }^{2}$ Discipline of Microbiology, School of Life Sciences, College of Agriculture, Engineering and Sciences, University of KwaZulu-Natal, Durban, South Africa}

\section{Video abstract}

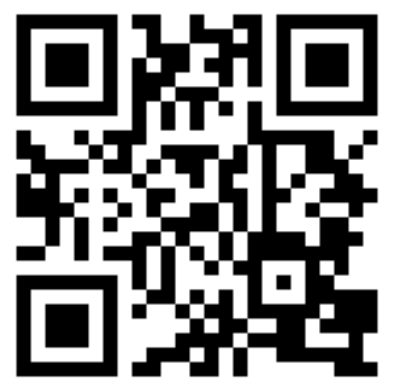

Point your SmartPhone at the code above. If you have a QR code reader the video abstract will appear. Or use: http://youtu.be/BODdLWISWrE

Correspondence: Mohamed E El Zowalaty

Virology and Microbiology Research Group, School of Health Sciences, Blok F2 Office 517, Westville campus, University Road, Private Bag X54004,

Durban 4000, South Africa

Tel +2731260789 I

$\mathrm{Fax}+27312607872$

Email elzowalatym@ukzn.ac.za

\begin{abstract}
Acinetobacter baumannii is a leading cause of nosocomial infections due to its increased antibiotic resistance and virulence. The ability of $A$. baumannii to form biofilms contributes to its survival in adverse environmental conditions including hospital environments and medical devices. A. baumannii has undoubtedly propelled the interest of biomedical researchers due to its broad range of associated infections especially in hospital intensive care units. The interplay among microbial physicochemistry, alterations in the phenotype and genotypic determinants, and the impact of existing ecological niche and the chemistry of antimicrobial agents has led to enhanced biofilm formation resulting in limited access of drugs to their specific targets. Understanding the triggers to biofilm formation is a step towards limiting and containing biofilm-associated infections and development of biofilm-specific countermeasures. The present review therefore focused on explaining the impact of environmental factors, antimicrobial resistance, gene alteration and regulation, and the prevailing microbial ecology in A. baumannii biofilm formation and gives insights into prospective anti-infective treatments.
\end{abstract}

Keywords: Acinetobacter baumannii, biofilm, physicochemical, antibiotics, resistance, in vivo model, virulence, treatment

\section{Introduction}

Acinetobacter baumannii belongs to the family Moraxellaceae in the class Proteobacteria of Eubacteria. ${ }^{1}$ The acceptable phylogeny, classification, and interpretation of the taxonomy of the genus, Acinetobacter, has been confused due to the myriads of classification systems established by scientists early on, ${ }^{2}$ with over 40 known species within the genus. ${ }^{3}$ The most clinically important Acinetobacter sp. is Acinetobacter baumannii, ${ }^{4}$ because of its association with hospital acquired-infections. ${ }^{5}$ Others include: Acinetobacter baylyi, Acinetobacter grimontii, Acinetobacter junii, and Acinetobacter calcoaceticus-A. baumannii (ACB) complex. ${ }^{6,7} A$. baumannii is a Gram-negative, nonmotile, strictly aerobic, non-fermentative, non-sporing, coccobacillus, which is notorious for its ability to survive in a variety of environmental conditions. ${ }^{8}$ The notorious bacterium A. baumannii has been referred to as "Iraqibacter" since its emergence in the US military treatment facilities when it caused serious problematic infections among soldiers during the Iraq and Afghanistan war-zone medical facilities. ${ }^{8}$ Hospital-acquired pneumonia, skin and soft tissue infections, and nosocomial meningitis are but a few infections caused by $A$. baumannii. ${ }^{9}$ It became a serious issue in medical treatment due 
to its exceedingly increased resistance to almost all known antibiotics and host immune responses. ${ }^{10}$ The possession of virulence factors such as the ability to form biofilm and survive in dry and desiccated environmental conditions have created challenges and epidemics in the health treatment, especially in critically ill patients in hospital intensive care units (ICUs). ${ }^{11}$ Here, we review the parameters that trigger biofilm formation with a view to understanding $A$. baumannii biofilm formation and also to assess the relationships between biofilm formation, virulence, and antimicrobial resistance.

\section{Search strategy}

We searched Academic Search Complete, CINAHL with Full Text, and PubMed (Medline) databases through Ebscohost and Google Scholar, for articles on A. baumannii biofilm published in English between the years 2008 and 2018, using the keywords "Acinetobacter baumannii", "biofilm", "antibiotic resistance", "physicochemical factors", "in vivo studies" "virulence", and "treatment". Articles on biofilms from bacteria other than A. baumannii and the dates stated were excluded except when necessary.

\section{Biofilm formation on biotic and abiotic surfaces}

Biofilms are aggregates of microbial cells that are surrounded by self-produced exopolysaccharide matrices on the surfaces of biotic or abiotic surfaces. Biofilms demonstrate greater protection against antibiotics, host immune defense, and adverse environmental conditions than the free-living cells. ${ }^{12}$ It is estimated that $65-80 \%$ of human infectious are caused by biofilm-forming bacteria. ${ }^{13}$ Biofilm-associated infections are chronic infections, hence, they require higher doses of antibiotics for treatment than planktonic cells' acute infection, with the resulting antimicrobial resistance having led to increased death, prolonged hospital stays, considerable economic loss, and loss of protection for patients. ${ }^{14}$ The factors that lead to enhanced antibiotic resistance in biofilm phenotype include impaired drug diffusion due to microbial aggregations, over-expression of the exopolymeric substance (EPS) matrix, alterations in microbial phenotypic and genotypic features due to stress responses, and physiological heterogeneity due to physicochemical gradients and persisters. ${ }^{15}$ The phenotypic and genotypic features are triggered when bacterial cells produce quorum sensing (QS) signals in a cell density-dependent manner which helps in cell-to-cell communication during changes in various environmental factors such as temperature, oxygen level, acidity, and the quality of growth medium. ${ }^{16}$ Stressors, including antibiotics when administered at concentrations below the minimum inhibitory concentration (MIC), also induce biofilm formation in a variety of bacterial species. ${ }^{17}$

It is highly likely that biofilm formation plays an important role in the interactions of $A$. baumannii with its host, and contributes to medical-device-associated infections. ${ }^{18} \mathrm{~A}$. baumannii demonstrates increased tolerance to extracellular stressors when part of biofilm communities. ${ }^{18}$ Based on a review of literatures, a number of factors: physicochemical and microbial (surface appendages, adhesins, capsular polysaccharides, virulence and resistance determinants), contribute to the formation and maintenance of A. baumannii biofilms.

\section{Ecology and its influence on the formation of biofilms by \\ A. baumannii}

Various ecological niches have been reported to support biofilm-forming A. baumannii. Bacterial cells of A. baumannii demonstrated strong biofilm formation in skin and soft-tissue infections, as well as in wounds and on occlusive dressings. ${ }^{18}$ A. baumannii biofilm communities were also observed on abiotic surfaces, including health care-associated equipment (endotracheal tubes), as well as polycarbonate and stainless steel. ${ }^{18}$

Bacterial isolates from urinary catheters, hospitalized patients in ICUs, and the environment are among the top biofilm producers. ${ }^{19,20}$ A. baumannii recovered from blood, urine, sputum, catheters, cerebrospinal fluid, bronchoalveolar lavage, exudates, and burned skin were equally shown to produce biofilms. ${ }^{21}$ Environmental isolates collected from patients' rooms at Federal University of Uberlandia Hospital in Brazil were also reported to produce biofilm. ${ }^{22}$ These may suggest that the source of microbial cells may have a great impact on the capacity of the cells to produce biofilm.

Studies have reported the influence of environmental factors, strain location, and ecological niche on the distribution and capacity of bacterial strains to produce biofilm. ${ }^{23}$ Although biofilm-producing bacteria exist in various environments and habitats, biofilm-forming A. baumannii exist almost exclusively in hospital environments. Within the same hospital, bacteria at a specific location may produce more biofilm than in another location. For instance, a statistically significant difference was observed between biofilm producers in surgical and medical wards $(P<0.05)$ and the groups $(P<0.05)$ despite the bacteria are of similar strain. ${ }^{23}$ Skin ulceration has been reported to harbor $A$. baumannii strains with biofilm-producing property which has been related to the major virulence factors, promoting bacterial persistence 
and chronicity in a separate and specific manner different from multidrug resistant (MDR) phenotypes. ${ }^{25}$

It was observed that transposition of insertion sequence (IS) elements influenced the ability of bacteria to overcome environmental challenges and adapt to new environmental niches, ${ }^{26,27}$ and this can, therefore, be a contributing factor to the existence of biofilm-specific strains in particular environments.

\section{Effect of polymicrobial existence}

Polymicrobial communities and associated genetic diversities create synergy within and among bacterial species during biofilm formation, with the polymicrobial existence of bacterial species therfore having significant implications for the clinical treatment of infections. ${ }^{28}$ Bacteria in polymicrobial communities produced more biofilms than when grown individually. A polymicrobial community of Pseudomonas and Enterococcus, Acinetobacter and Staphylococcus or Corynebacterium and Staphylococcus produced more biofilm than that formed by Enterococcus faecalis and Staphylococcus and E. faecalis and Corynebacterium. ${ }^{29}$ A study investigated the interspecies biofilm-forming abilities of Leptospira interrogans with 21 bacterial isolates belonging to ten genera. ${ }^{29}$ It was reported that though Azospirillum brasilense RMRCPB showed maximum interspecies co-aggregation with Leptospira strains ( $>75 \%$, visual score of +4$)$, isolates belonging to the genera Acinetobacter, Sphingomonas, Micrococcus, Brevundimonas, and Paracoccus formed significant biofilm and co-aggregation. ${ }^{29}$ Microbial species lacking in one or more enzymes necessary for metabolism, such as polysaccharide-degrading enzymes, may thrive in the presence of another bacterium producing such active enzymes during polymicrobial aggregation. For example, it was shown that biofilm formation by Acinetobacter oleivorans DR1, when a naphthalene-degrading Pseudomonas spp. AS1 was present, increased due to exopolysaccharides generated by the latter. ${ }^{30}$ A. oleivorans DR1, which could not grow due to its inability to degrade naphthalene, grew under naphthaleneamended conditions in the presence of naphthalene-degrading Pseudomonas sp. AS1. ${ }^{30}$ Transformation and conjugation experiments using A. baumannii harboring plasmid-mediated antibiotic resistant genes were used to demonstrate the possibility of intergeneric gene transfer and there was no difference observed between the wild-type and plasmid-cured strains with respect to their biofilm-forming ability. ${ }^{31}$

\section{Physicochemical factors affecting biofilm formation}

The physical and chemical alterations in the environment influence not only microbial phenotypic features but also the expression of important functions. ${ }^{32}$ Factors such as surface hydrophobicity, temperature, and oxygen concentration have been reported to influence biofilm formation in A. baumanni and other bacteria such as Clavibacter michiganensis. ${ }^{33,34}$ The general effects of the important physicochemical factors affecting $A$. baumannii biofilm formation are summarized in Table 1.

\section{Temperature}

The influence of temperature on the capacity of microbial cells to adhere to and form biofilms on surfaces has been widely reported. ${ }^{38,80}$ It was observed that Acinetobacter spp. biofilm formation was more prolific at $25^{\circ} \mathrm{C}$ than at $37^{\circ} \mathrm{C} .{ }^{37}$ However, the optimum temperature for biofilm formation by A. baumannii was reported to be $30^{\circ} \mathrm{C}$ at $\mathrm{pH} 7.0$ in a medium containing sodium chloride at a concentration of $5 \mathrm{gL}^{-1} \cdot{ }^{31}$ The factors that influenced biofilm formation of $A$. baumannii on medical devices in the ICU of an Algerian university hospital included temperature, hydrophobicity of the bacterial cell wall, duration of implantation, and nature of the medical device. ${ }^{40} \mathrm{~A}$ report demonstrated that $A$. baumannii strains formed biofilm better at $30^{\circ} \mathrm{C}$ than at $37^{\circ} \mathrm{C}$ while another showed that biofilm formation on plastic surfaces was high at $28^{\circ} \mathrm{C}$ due to the upregulation of certain biofilm-associated proteins (Baps) such as Csu and iron-uptake proteins in A. baumannii. ${ }^{39}$

\section{Growth media}

Biofilm formation of $A$. baumannii was reported to be influenced by growth conditions and environmental stress factors. ${ }^{48}$ The choice of growth medium for biofilm growth may depend on several factors such as the bacterial species and incubation conditions. For instance, A. baumannii cultured in glucose-based medium and exposed to sub-inhibitory concentrations of antibiotics such as imipenem can induce biofilm formation and lead to increased iron uptake in a clinical MDR phenotype. ${ }^{48}$ It was reported that there was a substantial inter-strain variability and growth medium-dependence for biofilm formation in A. baumannii from both veterinary and clinical sources. ${ }^{33}$ Different levels of biofilm growth were observed with five well-characterized $A$. baumannii strains cultured in three iron-poor media depending on the strain. ${ }^{33}$ Although all strains produced increased biofilm in Tryptic Soy Broth dialysate when compared with other iron-poor media, the report supports the notion that biofilm formation in $A$. baumannii is strain-dependent. ${ }^{33}$

Media that limit its growth, such as drinking water were reported to support biofilm-producing Acinetobacter species in water distribution systems. ${ }^{81}$ For instance, a study in Shanghai, China characterized the community diversity and abun- 
Table I The effects of physicochemical factors on biofilm formation in Acinetobacter baumannii

\begin{tabular}{|c|c|c|}
\hline $\begin{array}{l}\text { Physicochemical } \\
\text { factors }\end{array}$ & General effects & References \\
\hline Temperature & $\begin{array}{l}\text { High temperature enhances cell hydrophobicity which results in biofilm formation. } \\
\text { High temperature increases nutrient uptake which leads to increased biofilm formation. } \\
\text { Production of chaperon-usher assembly is high at } 28^{\circ} \mathrm{C} \text { in Acinetobacter baumannii } \\
\text { High temperature induces the production of polysaccharides. }\end{array}$ & $35-42$ \\
\hline $\begin{array}{l}\text { Type and nutrient } \\
\text { availability }\end{array}$ & $\begin{array}{l}\text { Concentration-dependent effect on biofilm accumulation. } \\
\text { Higher nutrient concentrations decrease biofilm accumulation due to detachment and reduced } \\
\text { competition among bacterial isolates. } \\
\text { Poor nutrient media increases biofilm production. } \\
\text { Presence of sucrose, calcium, and phosphate enhances biofilm formation. } \\
\text { Extreme nutrient limitation results in decreased exopolysaccharide synthesis and thus decreased } \\
\text { biofilm formation. } \\
\text { Influences metal-ion-mediated cross-linking required for EPS synthesis. }\end{array}$ & $41,43-49$ \\
\hline Growth surface & $\begin{array}{l}\text { Surface roughness shelters bacteria against shear forces, allows time for change to irreversible } \\
\text { attachment, and hence facilitates biofilm formation. } \\
\text { Smooth surface coating could lead to reduced biofilm formation. } \\
\text { Chemical and nutritional composition of organic surfaces promotes better bacterial adherence. } \\
\text { Surface charge and hydrophobicity influence biofilm formation in electroactive microbes. }\end{array}$ & $48,50-56$ \\
\hline $\begin{array}{l}\text { Iron } \\
\text { concentration }\end{array}$ & $\begin{array}{l}\text { Has a strain-dependent association with biofilm formation and type of iron source. } \\
\text { Iron limitation triggers up-regulation of Baps. } \\
\text { Higher iron concentration allows for increased resistance to certain antibiotics through signaling or } \\
\text { interacting with the antibiotics themselves. } \\
\text { Stimulates EPS production via repression of rhamnolipid production or inhibition of transcription } \\
\text { regulator that leads to alginate synthesis. } \\
\text { Iron limitation enhances acyl-homoserine lactone production. }\end{array}$ & $57-63$ \\
\hline $\begin{array}{l}\text { Antimicrobial } \\
\text { agents/ } \\
\text { Disinfectants }\end{array}$ & $\begin{array}{l}\text { Constitutes stress for enhanced induced gene regulation. } \\
\text { Offers fitness advantage for resistant strains, resulting in biofilm formation. } \\
\text { Hydrophilic antimicrobial coating repels bacterial adhesion, reduces biofilm formation. }\end{array}$ & $64-66$ \\
\hline $\begin{array}{l}\text { Agitation } \\
\text { Quorum sensing } \\
\text { molecules }\end{array}$ & $\begin{array}{l}\text { Flow movement influences the composition and cohesiveness of the EPS matrix formed during biofilm } \\
\text { production. } \\
\text { Influences the composition and physical properties by affecting the elasticity of the biofilm. } \\
\text { It modulates cell functions such as pathogenesis, nutrient uptake, motility, cell communication, and } \\
\text { production of secondary metabolites. }\end{array}$ & $67-69$ \\
\hline $\begin{array}{l}\text { Electrostatic } \\
\text { interactions }\end{array}$ & $\begin{array}{l}\text { Bacteria attach rapidly and tightly to positively charged surfaces. } \\
\text { Electrostatic repulsion disturbs cell contact with negatively charged surfaces. }\end{array}$ & $74-76$ \\
\hline $\begin{array}{l}\mathrm{pH} \text { and ion } \\
\text { content }\end{array}$ & $\begin{array}{l}\text { Divalent cationic ions enhance bacterial attachment to surfaces. } \\
\text { Cations stabilize the interaction between negative charge surface and anionic substrates. }\end{array}$ & $77-79$ \\
\hline
\end{tabular}

Abbreviation: EPS, exopolymeric substance.

dance of biofilms in a full-scale drinking water distribution system by denaturing gradient gel electrophoresis and analysis of the 16S rRNA sequences with heterotrophic plate count methods. It was observed that Acidovorax, Ralstonia and Acinetobacter were the most predominant biofilm-forming species. ${ }^{82}$ Among other bacterial species, A. baumannii was the most common species in biofilms formed in drinking water pipe systems. ${ }^{83}$ Though $A$. baumannii persists and prospers in very diverse ecological niches including medical environments, it was demonstrated that biofilm formation is not important for the epidemic spread of $A$. baumannii. $^{84}$

\section{Surfaces}

The biofilm-forming abilities of Acinetobacter species, both at air-liquid and solid-liquid interphases, and at different temperatures were compared.$^{37}$ It was observed that not only did isolates belonging to ACB-complex form biofilm at the air-liquid interface, but also demonstrated greater biofilm formation than other species at solid-liquid interfaces.$^{37}$ In addition, within the complex, biofilm formation by A. baumannii and Acinetobacter G13TU was four times greater than that of Acinetobacter G3, ${ }^{37}$ confirming the former as a higher biofilm-producing species of the genus Acinetobacter.

A. baumannii has the ability to form biofilms on different surfaces and develop high biomass biofilm on stainless steel, polystyrene, and polycarbonate, a thermoplastic material that is often used to construct medical devices. ${ }^{53}$ Although A. baumanni can form biofilm on most abiotic surfaces, clinical isolates have preference for polypropylene surfaces ${ }^{85} \mathrm{~A}$ 
comparative study on the ability of $A$. baumannii to produce biofilm on two different surfaces, ie, polycarbonate and polypropylene, revealed that $A$. baumanni isolates formed more biofilm on polypropylene under agitation than on the polycarbonate surfaces. ${ }^{85}$ It was demonstrated that $A$. baumannii isolated from wound cultures formed strong biofilm on stainless steel surfaces. ${ }^{54}$ The ability of $A$. baumanni to form matured biofilms on polypropylene, polystyrene, titanium, and other medical-associated devices has been associated with several factors including the presence of Bap. ${ }^{55,56}$ The extent to which these factors promote biofilm production, especially in the human cell environment, is not clearly understood. However, certain studies have shown that these factors may have variable influence on different microbial genera and among microbial species in epithelial cells. For instance, a study reported that the capacity for biofilm formation and the clinical success of A. baumannii on epithelial cells varied between the different A. baumannii strains. ${ }^{86}$ This is probably due to the extent of the contribution of diverse factors including Bap to inflammatory cytokine induction by different strains of $A$. baumannii. ${ }^{87}$

\section{Iron concentration and QS}

Iron limitation enhances the expression of the QS genes which control density-dependent virulence factors in microbial species. ${ }^{88}$ QS signaling molecules in some bacterial species such as Pseudomonas aeruginosa are strong chelators of iron, which causes limitation of iron leading to a stress response. ${ }^{63}$ Besides iron chelation, siderophores are reported to have non-classical functions such as antibiotic activity and act as zincophores, chalkophores and metallophores for other metals, metal toxin sequester and oxidative stress. ${ }^{89}$

A. baumannii has one QS system that makes use of the AbaR receptor protein and $N$-(3-hydroxydodecanoyl)-1homoserine lactone, thereby regulating virulence factors such as biofilm formation and surface motility. ${ }^{70,71}$ The influence of iron-III limitation on $N$-acyl homoserine lactone (AHL), siderophore, and catechol on the virulence factors of $\mathrm{A}$. baumannii biofilm-forming clinical strains was investigated. ${ }^{59} \mathrm{~A}$ higher amount of AHL (70\%) was detected in the presence of low concentrations of iron-III $(20 \mu \mathrm{M})$. Furthermore, screening for $l u x I$ and $l u x R$ showed that the presence of both genes was required in the isolates for high AHL activity. ${ }^{59}$ Both AHL production and biofilm formation were found to be regulated by iron concentration in a dose-dependent manner. ${ }^{59}$ Hence, biofilm-formers including $A$. baumannii persist in limited iron environments. ${ }^{59}$ Analysis of bap expression by real-time reverse transcription polymerase chain reaction (RT-qPCR) showed that some tested $A$. baumannii isolates had four-fold bap over-expression in the presence of low iron concentration $(20 \mu \mathrm{M}) .{ }^{90}$ However, the study showed that iron starvation was not the only factor required to stimulate biofilm production in A. baumannii, but that its formation was affected by growth medium and strain identity. ${ }^{33}$

\section{Virulence determinants in A. baumannii}

Microbial functions such as cell communication, surfaceregulated attachments, and secretion of macromolecules are essential factors for biofilm formation. ${ }^{91,92}$ A. baumannii expresses several virulence genes which make it a successful pathogen in humans and animals. ${ }^{93}$ Some of these factors such as $\mathrm{K} 1$ capsular polysaccharides, surface antigen protein 1, iron acquisition systems, outer membrane porins, acinetobactin transporters together with resistance to the majority of antibiotics have made $A$. baumannii an increasingly important pathogen. ${ }^{93}$ A . baumannii harbors repertoires of biofilm-related virulence genes and proteins which contribute to its ability to adhere and form biofilms on biotic and abiotic surfaces. ${ }^{96}$ The presence of these genes makes $A$. baumannii remain viable in diverse environments and a formidable force against antimicrobials and immune cells. ${ }^{97}$ These multiple genetic elements are controlled and regulated by complex regulatory networks, including those based on the presence of antibiotic resistance genes, environmental conditions, or cell density. The virulence determinants associated with biofilm and biofilm-specific resistance in A. baumannii are summarized in Table 2. Although factors that contribute to biofilm formation seem to be strain-dependent, ${ }^{93}$ some common factors have been identified.

\section{Bap}

Bap (biofilm-associated protein) is required for the threedimensional structure tower and water channel formation in biofilm. ${ }^{95}$ Bap is a cell-surface protein identical to the Staphylococcus Bap and was first identified in A. baumannii by Loehfelm et al. ${ }^{90}$ Bap, which is secreted via a type I secretion system, mediates $A$. baumannii biofilm formation and maturation. ${ }^{93}$ Bap plays a role in cell-cell adhesion and is required for the development of higher-order structures on medically relevant materials, such as polystyrene and titanium. ${ }^{90}$

Although most sequenced strains of $A$. baumannii carry a bap gene, many seem to have disrupted or truncated bap sequences, which might be attributed to recombination events or sequence alignment errors that are common to the highly repetitive elements of bap coding sequences. ${ }^{56}$ bap has been shown to express a highly variable protein. 
Table 2 Gene determinants associated with virulence and antibiotics resistance in Acinetobacter baumannii

\begin{tabular}{|c|c|c|c|}
\hline $\begin{array}{l}\text { Virulence } \\
\text { determinants }\end{array}$ & Type/Designation & Functions & References \\
\hline Bap & A bap & $\begin{array}{l}\text { Required for the three-dimensional structure tower } \\
\text { and water channel formation in biofilm }\end{array}$ & $60,90,135$ \\
\hline AdeABC & $\begin{array}{l}\text { AdeA - outer membrane protein associated with } \\
\text { carbapenem resistance } \\
\text { AdeB - an efflux pump membrane transporter } \\
\text { AdeC - outer membrane factor of the AdeABC } \\
\text { multidrug efflux complex }\end{array}$ & $\begin{array}{l}\text { Involved in multidrug resistance and biofilm } \\
\text { formation }\end{array}$ & $98,99,100,101$ \\
\hline AdeFGH & $\begin{array}{l}\text { A resistance-nodulation-cell division (RND) } \\
\text { antibiotics efflux system }\end{array}$ & $\begin{array}{l}\text { Involved in synthesis and transport of auto-inducer } \\
\text { molecules } \\
\text { Involved in multidrug resistance }\end{array}$ & 127,142 \\
\hline AdelJK & An RND antibiotics efflux system & Involved in multidrug resistance & \\
\hline PgaABCD & $\begin{array}{l}\text { Outer membrane secretion involved in biogenesis } \\
\text { of poly-beta-I, 6-N-acetyl-D-glucosamine }\end{array}$ & $\begin{array}{l}\text { Substrate-specific transmembrane transporter } \\
\text { activity and also involved in biofilm formation }\end{array}$ & 102 \\
\hline CsuA/ & A member of usher-chaperone fimbriae & Pili-biogenesis and biofilm formation & 103 \\
\hline BABCDE & $\begin{array}{l}\text { CusD - a member of the usher operon involved in } \\
\text { outer membrane protein }\end{array}$ & & \\
\hline AbalR & A quorum sensing system & $\begin{array}{l}\text { Regulates factors such as biofilm formation and } \\
\text { surface motility }\end{array}$ & 154,155 \\
\hline OmpA/M & $\begin{array}{l}\text { Outer membrane protein which maintains cell } \\
\text { membrane integrity and enhances cell adhesion to } \\
\text { surfaces }\end{array}$ & $\begin{array}{l}\text { Necessary for antibiotic resistance, biofilm } \\
\text { formation; modulates biogenesis of outer membrane } \\
\text { vesicles }\end{array}$ & 104,105 \\
\hline OprB/C & $\begin{array}{l}\text { Porin proteins which maintain cell membrane } \\
\text { integrity and functions }\end{array}$ & Involved in antibiotic efflux system & 106 \\
\hline SmpA & $\begin{array}{l}\text { Belongs to the SmpA/OmpA family protein } \\
\text { domain in the fgr } 9 \text { which enhances adhesion and } \\
\text { membrane integrity in bacteria. }\end{array}$ & Vital for adhesion and complement evasion & 107,108 \\
\hline CarO & A membrane fusion protein & $\begin{array}{l}\text { Involved in carbapenem resistance. Also essential for } \\
\text { L-ornithine uptake }\end{array}$ & 94,109 \\
\hline Pili & A type IV pili system & Required for twitching motility & 18 \\
\hline EpsA & A capsular polysaccharide protein & $\begin{array}{l}\text { Putative polysaccharide export outer membrane } \\
\text { protein } \\
\text { Involved in } \mathrm{KI} \text {-capsular poly saccharide biogenesis }\end{array}$ & 110 \\
\hline Ptk & Putative protein tyrosine kinase & Involved in KI-capsular poly saccharide biogenesis & 110 \\
\hline TssB (VipA) & VI polysaccharide biosynthesis protein & Involved in inter-bacterial competition and responses & 111 \\
\hline Wzx & Transport protein in $\mathrm{K}$ locus & $\begin{array}{l}\text { Involved in the synthesis assembly and translocation } \\
\text { of oligosaccharides repeat units }\end{array}$ & 112,113 \\
\hline GntK & Thermo-resistant gluconokinase & Regulation of stress responses & 114 \\
\hline Lux & Quorum sensing gene & $\begin{array}{l}\text { Regulates cell-to-cell communication necessary for } \\
\text { signaling during biofilm formation }\end{array}$ & $26,71,105$ \\
\hline PilA & Type IV pili gene & $\begin{array}{l}\text { Involved in twitching motility and surface-associated } \\
\text { motility }\end{array}$ & 92 \\
\hline BarA & GacS-like sensor kinase & Involved in transportation of iron and nutrients & 115 \\
\hline BarB & $A B C$ transporter & Involved in nutrient and iron transportation & 115 \\
\hline BasC & Probable acinetobactin biosynthesis protein & $\begin{array}{l}\text { Involved in cellular transportation of iron and } \\
\text { nutrients }\end{array}$ & 115 \\
\hline BasD & Non-ribosomal peptide synthetase & Involved in iron acquisition in iron limiting condition & 116 \\
\hline BauA & Ferric acinetobactin receptor & Associated with siderophore-mediated iron uptake & 117,118 \\
\hline AmpC & AmpC $\beta$-lactamase & Present in a form of ADC type AmpC genes & 119,120 \\
\hline Bla-OXA-69 & Carbapenem-hydrolyzing oxacillinase & Antibiotic resistance & 121,122 \\
\hline Bla-OXA-75 & Carbapenem-hydrolyzing oxacillinase & Antibiotic resistance & 122 \\
\hline MVim & A metallo-beta-lactamase protein & Involved in multidrug resistance & 121,123 \\
\hline
\end{tabular}

Partial sequencing of bap performed on the index case strain MS1968, revealed that bap is a large and highly repetitive gene of approximately $16 \mathrm{~kb}$ in size. ${ }^{56}$ The fully sequenced open reading frame was $25,863 \mathrm{bp}$ and it encoded a protein with a predicted mass of $854 \mathrm{kDa} .{ }^{56}$ Bap could be identified through a repetitive structure identical to a bacterial cell 
surface adhesion protein. Using phylogenetic analysis, it was shown that bap from strain MS1968 clusters with bap sequences from clonal complex 2 (CC2) strains ACICU, TCDC-AB0715, and 1656-2, were distinct from bap in CC1 strains, ${ }^{56}$ revealing that bap was clonal. Further analysis of the bap-positive strain showed that Bap is expressed at the cell surface and that it is associated with biofilm formation and could be inhibited with affinity-purified antibodies. ${ }^{56}$ Time course confocal laser scanning microscopy and threedimensional image analysis were used to observe that $A$. baumannii mutant in the coding sequence of bap was unable to sustain biofilm thickness and volume. ${ }^{90}$

The prevalence, expression, and function of Bap in 24 carbapenem-resistant A. baumannii ST92 strains isolated from a single institution were previously investigated ${ }^{56}$ Out of the 24 strains, 22 expressed Bap. The biofilm-forming ability of 65 MDR $A$. baumannii isolates and its association with biofilm-related genes was assessed using RT-qPCR. oтp $A$ and $\operatorname{csu} E$ genes were detected in all isolates, however, bap and blaPER-1 were detected in 43 (66\%) and 42 (64\%) of the phenotypes that showed strong and moderate biofilm activities $(P \leq 0.05)$, respectively. ${ }^{90}$ It was found that the frequency of genes related to biofilm formation in 27 A. baumannii isolates were ompA (100\%), bap (30\%), and blaPER-1 (44\%). ${ }^{124}$ Western blotting revealed that all the isolates expressed OmpA and only eight isolates were positive for Bap factor. ${ }^{124}$ Double-negative bap/blaPER-1 isolates were recovered from bloodstream infections and had low biofilm-forming capability, unlike the clinical isolates from throat infections which were blaPER-1-positive and had a high biofilm forming ability. ${ }^{124}$

\section{Efflux systems}

Efflux systems in A. baumannii are chromosomally-encoded, two component efflux systems and are responsible for resistance to several microbicides when over-expressed. ${ }^{125}$ The genes encoding these systems are carried by various genetic elements such as tet $A$ and $c m l A$ efflux pumps which are also responsible for tetracycline and chloramphenicol resistance. ${ }^{126}$ There are five subfamilies of efflux systems which play crucial roles in antibiotic resistance: ABC transporters, SMR, MATE, MFS, and RND families. ${ }^{127}$ Among these five "superfamilies", RND efflux systems are the most prevalent in MDR A. baumannii and have been reported to play a role in the efflux of a wide range of substrates which include antibiotics, dyes, biocides, detergents, and antiseptics. ${ }^{127}$ There are three types of RND pumps that are associated with $A$. baumannii, these being, AdeABC, AdeFGH, and AdeIJK.

The AdeABC (Acinetobacter drug efflux) pump comprises three components: an inner membrane pump (AdeA) which links the major fusion protein to the outer membrane factor (AdeC). ${ }^{127}$ The operon that encodes these systems is only expressed by MDR $A$. baumannii phenotype isolates when the efflux pump is over-expressed..$^{99}$ AdeABC is tightly regulated by AdeR-AdeS which is encoded by the adeRS operon and contributes to the acquisition of antibiotic resistance when over-produced in A. baumannii. ${ }^{99}$ Over-expression of Ade $\mathrm{ABC}$ contributes largely to multidrug resistance, decreased biofilm production, and altered membrane composition in A. baumannii. ${ }^{99}$ Aminoglycosides, beta-lactams, fluoroquinolones, tetracycline, tigecycline, macrolides, chloramphenicol, and trimethoprim resistant phenotypes are associated with mutations in the adeRS operon. ${ }^{128}$ AdeRS has been linked to the capacity of $A$. baumannii to produce biofilm. ${ }^{101}$ It was reported that inactivation of adeRS led to the loss of biofilm formation in an A. baumannii isolate due to decreased expression of adeABC, pill, com, and a pgaClike gene, which influenced its antimicrobial resistance and virulence interactions. ${ }^{101}$ Deletion of $a d e A B$ influenced the ability of $A$. baumannii to form biofilms on plastic, in a strain-specific manner. ${ }^{101}$ It was observed that increasing amounts of microbicides alone or in combination resulted in the modification of expression, and function of $a d e B$ gene of the RND-type efflux pump AdeABC and hence, the destruction of the biofilm mass and viability. ${ }^{129}$

AdeFGH is proposed to be regulated by a LysR-type transcriptional regulator and confers multidrug resistance in A. baumannii when over-expressed. ${ }^{127}$ The AdeFGH efflux pump is linked to the synthesis and transport of autoinducer molecules during $A$. baumannii biofilm formation. ${ }^{130}$ Up-regulation of ade $G$ correlated with biofilm induction especially when treated with low-dose antimicrobial therapy such as fluoroquinolone. ${ }^{130}$ However, the level of gene transcription varied between bacterial genotypes, antibiotics, and antibiotic concentrations. ${ }^{130}$ It was reported that the downregulation of efflux pumps, especially the AdeFGH efflux pump resulted in a reduction in A. baumannii biofilm in the presence of sub-inhibitory concentrations of tigecycline. ${ }^{125}$ When the effect of sub-inhibitory tigecycline concentrations was investigated using RT-qPCR, a positive correlation was established between biofilm formation induced by sub-inhibitory concentrations and the expression of biofilm-associated genes, especially AdeFGH efflux pump genes. ${ }^{125}$ 
AdeIJK is intrinsic to A. baumannii and contributes to natural resistance when over-expressed above a certain threshold. ${ }^{128,131}$ It is the second RND type efflux pump in A. baumannii and comprises adeI, adeJ, and adeK genes. ${ }^{132}$ The AdeIJK pump contributes to resistance to beta-lactams, chloramphenicol, tetracycline, erythromycin, lincosamides, fluoroquinolones, fusidic acid, novobiocin, rifampin, trimethoprim, acridine, safranin, pyronine, and sodium dodecyl sulfate. ${ }^{128}$ It was suggested that over-expression of AdeIJK contributes largely to multidrug resistance, decreased biofilm production, and altered membrane composition in $A$. baumannii. ${ }^{99}$

There are other non-RND efflux systems, such as CraA, AmvA, AbeM, and AbeS which have also been characterized for A. baumannii, while AdeXYZ and AdeDE efflux pumps have been characterized for other Acinetobacter spp., ${ }^{127}$ but have not been implicated in biofilm formation.

\section{PgaABCD gene clusters}

The pgaABCD gene cluster encodes poly-(1-6)-Nacetylglucosamine (PNAG), one of the most important structures for biofilm formation in both Gram-negative and Gram-positive microorganisms. ${ }^{102}$ The association of the expression of pgaABC gene clusters and altered biofilm phenotypes was investigated. ${ }^{131}$ It was observed that the expression of pgaB in a clinical $A$. baumannii strain was much higher than that of a standard strain, and was associated with an increase in the capacity and thickness of biofilm formation. ${ }^{133}$ The influence of altered expression of the QS gene abaI and pgaB in A. baumannii strains isolated from burn patients on biofilm formation, extracellular matrix expression, and drug resistance was investigated using RT-qPCR. ${ }^{133}$ The relative expression of $a b a I$ and pgaB in the MDR strain was much higher than that of a drug-sensitive strain. ${ }^{133}$ The biofilm thickness of the MDR strain was thicker than that of the standard strain. ${ }^{133}$ The increased expression of QS abaI in the drug-resistant $A$. baumannii strains may have up-regulated the expression of pgaB, which possibly leads to increased production of extracellular matrix and hence increased biofilm formation and enhanced drug resistance in A. baumannii. ${ }^{133}$ Another study aimed to confirm if the pgaABCD locus was responsible for cell-associated PNAG and to investigate its involvement in biofilm production. ${ }^{102}$ It was revealed that not only did the deletion of pgaABCD result in an absence of PNAG, but also led to the loss of the strong biofilm phenotype, which was restored after complementation. ${ }^{102}$

\section{CsuA/BABCD chaperone-usher pilli assembly system}

The majority of $A$. baumannii strains encode and produce a type I chaperone-usher pilus system designated Csu pili. ${ }^{18}$ Csu pili, which are regulated by the BfmRS two-component regulatory system, ${ }^{147}$ are not required for association with biotic surfaces, such as human epithelial cells, ${ }^{87}$ but are critical for biofilm formation and maintenance on abiotic surfaces, including polystyrene.

Although many $A$. baumannii strains seem to carry the csuA/BABCDE locus, a subset of clinical isolates have lost the $c s u$ cluster. ${ }^{134}$ This might suggest that these pili may not be required for biofilm formation and maintenance in all strains or that other pili systems may functionally replace them. ${ }^{134}$ A second two-component system termed GacSA was shown to moderately control $c s u$ gene expression and thus indirectly affects biofilm formation..$^{93}$ The expression of Csu pili in A. baumannii is completely repressed by subinhibitory concentrations of trimethoprim-sulfamethoxazole, which is indicative that improper use of antibiotics can alter population-level behaviors and may promote a planktonic lifestyle. ${ }^{93}$

QS molecules have been linked with up-regulation in gene expression of the $b f m S$ and $b f m R$ system, which has been predicted to contribute to the enhanced biofilm-forming capacity of strains on abiotic surfaces. ${ }^{135}$ The possible role of QS signaling molecules to regulate pili formation and the ability of A. baumannii to form biofilms on abiotic surfaces was investigated. ${ }^{136}$ Using transmission electron microscopy analysis, cells cultured in Mueller-Hinton broth in the presence of $N$-hexanoyl-L-homoserine lactone (C6-HSL) showed more abundant pili-like structures than the control strain. ${ }^{136}$ In another knock-out and complementation study, the role of the response regulator component of the two-component system and sensor kinase, $b f m S$, in the type strain $A$. baumannii ATCC 17978 was investigated. ${ }^{137}$ The $b f m S$ knock-out mutant showed a drastic reduction in biofilm formation, lost its adherence to eukaryotic cells, and demonstrated greater sensitivity to serum killing compared with the parental strain. ${ }^{137}$ Proteomic analysis also revealed that the release of outer membrane proteins (OMPs) including CarO and OmpA, was associated with the transposon-mediated inactivation of bfmS in A. baumannii strain. ${ }^{137}$

A biofilm hyper-producing $A$. baumannii strain (MAR002) was investigated to identify the role of the chaperone-usher pili assembly systems in pilus biogenesis pathways and the ability to form biofilm and attachment to 
A549 human alveolar epithelial cells. ${ }^{138}$ Putative proteincoding genes involved in pili formation were identified based on the newly sequenced genome of MAR002 strain (JRHB01000001/2 or NZ_JRHB01000001/2). ${ }^{138}$ When assessed with RT-qPCR, LH92_11085 gene which belongs to the operon, LH92_11070-11085, was over-expressed ( 25 -fold more) in biofilm-associated cells compared to exponential planktonic cells. ${ }^{139}$ Transmission electron microscopy analysis of the LH92_11085 mutant showed the absence of long pili formations normally present in the wild-type. ${ }^{139}$

\section{AbalR QS system}

Co-colonization of same infection sites by A. baumannii and $P$. aeruginosa have been observed to employ AHL-based QS systems that co-ordinate biofilm the formation and coinfection of both species resulting in increased severity of the infection. ${ }^{140}$

$a b a I$ and $a b a R$ are QS genes expressed in A. baumannii but acquired horizontally from Halothiobacillus neapolitanus. ${ }^{141}$ This QS system is the only one in A. baumannii and involves the AbaR receptor protein which forms a complex with the AbaI (auto-inducer synthase)-generated N-(3. hydroxydodecanoyl)-L-homoserine lactone that regulates virulence factors such as biofilm formation and surface motility. ${ }^{142}$

It was found that biofilm formation among clinical isolates of Acinetobacter spp. was under the control of an auto-inducing QS molecule. ${ }^{143}$ The auto-inducer synthase gene, abaI, was present among the isolates that produce QS signaling molecules and a mutation in abal influenced Acinetobacter spp. biofilm-forming capabilities. ${ }^{143}$ The association between drug-resistance and the influence of $a b a R$ on biofilm formation of $A$. baumannii was investigated. ${ }^{160} \mathrm{It}$ was found that the abaR in A. baumannii was associated with biofilm formation. ${ }^{160}$ The abaR gene sequence of $A$. baumannii, ATCC 17978, was compared with aqsR (the LuxR-type receptor) of $A$. oleivorans $\mathrm{DR} 1$ and it was observed that the similarity to LuxR-type receptor was $80 \%$. ${ }^{144,145}$

QS molecules have been linked to up-regulation of the expression of $b f m S$ and $b f m R$ genes, thereby enhancing the ability of $A$. baumannii to form biofilm on abiotic surfaces. The possible role of QS signaling molecules in regulating pili formation and its ability in A. baumannii to form biofilms on abiotic surfaces was identified using transmission electron microscopy. ${ }^{136}$ There were more abundant pili-like structures in the co-cultured cells than the control strain. ${ }^{136}$
Another study linked the AdeFGH efflux pump to the synthesis and transport of auto-inducer molecules during biofilm formation. ${ }^{130}$ According to the study, up-regulation of ade $G$ was correlated with biofilm induction, especially when $A$. baumannii was treated with low-dose antimicrobial therapy such as fluoroquinolone antibiotics. ${ }^{130}$

\section{Porins}

Porins play a very important role in microbial virulence through drug exclusion mechanisms across the outer membrane channels. ${ }^{106} \mathrm{OmpA}$ is a prominent porin in $A$. baumannii and contributes to drug resistance, adhesion to epithelial cells, and biofilm formation. ${ }^{146}$ Two-dimensional gel electrophoresis revealed that OMP receptors were upregulated during biofilm formation. ${ }^{147}$

Several novel proteins such as CarO, OprD-like, DcaPlike, PstS, LysM, and Omp33, and histidine metabolism (like urocanase) were involved in biofilm formation in $A$. baumannii ${ }^{148}$ and its reduced drug permeability. ${ }^{104}$ It was reported that OMPs such as $\mathrm{CarO}$ and OmpA can interact physically with OXA-23 carbapenemase which can lead to enhanced antibiotic resistance. ${ }^{185}$

\section{Other biofilm-associated genes}

$\operatorname{alg} C$ is a biofilm-associated gene that mediates the synthesis of bi-functional enzyme, phosphomannomutase/ phosphoglucomutase, which is required to produce exopolysaccharide alginate and lipopolysaccharide core during biofilm formation. ${ }^{144}$ It was reported that the expression level of $\operatorname{alg} C$ in the MDR strain of $A$. baumannii was up-regulated in a biofilm mode of growth compared to a planktonic mode. ${ }^{144}$ Characterizing the quantitative gene expression pattern (using the relative quantification method) in real-time PCR revealed that biofilm cells showed an 81.59-fold expression compared with a 3.24-fold in planktonic cells $(p<0.05){ }^{144}$

It was found that the $A$. baumannii virulence factor, associated with LHp2_11085 gene is required for biofilm formation on abiotic structures and attachment to epithelial cell surfaces. ${ }^{145}$ However, validation of this is suggested through an in vivo infection model such as the larvae of the wax moth Galleria mellonella, which allows the study of $A$. baumannii pathogenesis and therapeutics. ${ }^{145}$ When validated, the protein encoded by the LHp2_11085 gene of MAR002 strain could be a target for therapeutic development. ${ }^{145}$

A recent study linked the A. baumannii ATCC 17978 A1S_0112-A1S_0119 gene cluster to biofilm formation and 
surface motility. ${ }^{149}$ Using microscopy, it was observed that a derivative lacking these genes was unable to form matured biofilm. ${ }^{149}$ The A1S_0114 gene is involved in expression of genes coding for surface proteins and pili-assembly systems. ${ }^{150}$ Experimental animal models, including vertebrate and invertebrate hosts, were used to demonstrate that A1S_0114 gene has a significant role in A. baumannii virulence. ${ }^{150}$ It was found that the A1S_0114 gene was necessary for proper attachment to A549 cells. ${ }^{150}$

\section{Antibiotic resistance and the A. baumannii biofilm phenotype}

A. baumannii is resistant to several classes of currently available antibiotics including $\beta$-lactams, aminoglycosides, cefotaxime, tetracycline, erythromycin, chloramphenicol, trimethoprim, and fluoroquinolones. ${ }^{11}$ MDR strains are often isolated from immuno-compromised patients and those treated with a broad spectrum of antibiotics. ${ }^{151}$

The type of association that exists between biofilm formation and antibiotic resistance phenotypes remains controversial. According to some studies, the type and the reservoir of resistance determinants harbored by $\mathrm{Aci}$ netobacter spp. can influence its capacity to form biofilm. Biofilm formation is more strongly associated with MDR A. baumannii strains than with the susceptible strains. ${ }^{21,152}$ A study performed on clinical isolates from patients with nosocomial infections in three hospitals in Tehran showed that at least $92 \%$ of the biofilm-forming isolates were MDR. ${ }^{21}$ A significant correlation between multidrug resistance and biofilm formation of clinical and environmental isolates was established, ${ }^{152}$ ie, clinical isolates had a higher ability to form strong biofilm than environmental isolates. ${ }^{152}$ However, in vitro susceptibility can be influenced by the method used. For instance, when the resistance profiles of 65 clinical Acinetobacter spp. strains were assessed by both disk diffusion and minimum inhibitory concentration (MIC) methods, 46\% were resistant by the MIC method compared with $32.3 \%$ using the disk diffusion assay. ${ }^{153}$ When the biofilm-forming ability was tested using the microtiter plate method, seven strains were strong biofilm producers, 18 were moderate biofilm producers, 20 were weak biofilm producers, and 20 were non-biofilm producers. ${ }^{153}$ A statistically significant association was observed between biofilm formation and imipenem resistance. ${ }^{153}$ It was found that $25 \%$ of the 72 clinical isolates of $A$. baumannii isolated from India were resistant to ampicillin-sulbactam, $36.1 \%$ to imipenem, $66.6 \%$ to ceftazidime, $72.2 \%$ to ciprofloxacin,
$80.5 \%$ to amikacin, and $84.7 \%$ to piperacillin while $62.5 \%$ $(45 / 72)$ of isolates produced biofilm. ${ }^{154}$ They established that the correlation between antibiotic resistance and biofilm formation was significant $(P=0.0004) .{ }^{154}$ Biofilm formation in clinical isolates of Acinetobacter spp. from intensive and non-intensive tertiary care hospital units in Bangladesh and its correlation with antibiotic resistance were reported. The majority of the clinical Acinetobacter spp. isolates were reported as MDR and biofilm producers, especially those isolated from ICU samples. ${ }^{155}$ More than $86 \%$ of the isolates from a tertiary care hospital in Mexico were resistant to ciprofloxacin, ceftazidime, and cefotaxime, and $50.7 \%$ and $35.5 \%$ of the isolates were resistant to imipenem and meropenem, respectively. ${ }^{156}$ Of these isolates, $28.3 \%$ and $25.7 \%$ were positive for the blaOXA-58 and blaOXA-72 genes, respectively. ${ }^{156}$ The report also associated biofilm production with resistance to imipenem $(P=0.002) .{ }^{156} \mathrm{It}$ was observed that drug-resistant $A$. baumannii associated with burn wounds readily formed biofilms, preventing antibacterial activity of topical agents mafenide acetate and chlorhexidine acetate commonly used for burn treatment. ${ }^{157}$ The combined use of ambroxol (secretolytic agent used in the treatment of respiratory diseases associated with viscid or excessive mucus) with topical agents provided a synergistic effect, with increased killing of wound-associated $A$. baumannii in biofilms. ${ }^{157}$ It was observed that $A$. baumannii isolated from sputum of elderly patients were all biofilmformers and showed different levels of antibiotic resistance, while strong biofilm formation correlated with resistance to gentamicin, minocycline, and ceftazidime $(P<0.05) .{ }^{158} \mathrm{It}$ was demonstrated that 12 out of 17 clinical $A$. baumannii isolates from a Portuguese hospital demonstrated multidrug resistance, while $74.7 \%$ of the MDR isolates showed biofilm forming ability. ${ }^{23}$ The majority of the isolates from urine samples showed resistance to gentamicin, tobramycin, and trimethoprim/sulfamethoxazole and readily form biofilm compared to susceptible strains and those from other sources. ${ }^{23}$ However, no statistical significance was observed between the ability to form biofilms and antibiotic resistance. Of the 100 clinical A. baumannii isolates examined in Korea, $77 \%$ exhibited enhanced biofilm formation relative to the $A$. baumannii strain type ATCC $19606 .{ }^{66}$ A. baumannii strains that exhibited increased biofilm formation showed greater differences in their MICs and minimal biofilm-inhibitory concentrations (MBIC) of colistin. ${ }^{62}$ While most isolates were susceptible to colistin when in the planktonic state, MBIC was $64 \mathrm{mg} / \mathrm{mL}$ when in the biofilm state. Thus, when 
growing in a biofilm, a much higher bactericidal concentration of colistin is required. ${ }^{62}$

A strong biofilm-forming MDR A. baumannii strain collected from a Chinese hospital showed that resistance to levofloxacin, cefepime, and gentamicin significantly decreased when biofilm-forming ability was strong. ${ }^{159}$ Biofilm-related genes such as bap, $b f$, and intI1 were observed to contribute to biofilm formation. ${ }^{150}$ Biofilm-forming A. baumannii strains were associated with reduced resistance to imipenem and ciprofloxacin with such strains possibly being less virulent. ${ }^{160}$ Most of the non-biofilm forming isolates were associated with ciprofloxacin resistance, ICU treatment, and isolated from respiratory tract infections while biofilm-formers were all associated with catheter-related infections, blood stream infections, and previous aminoglycoside use. ${ }^{160}$ This explains the view that biofilm-forming bacterial strains do not depend on antibiotic resistance and colonization characteristics as do non-biofilm formers for their survival in a hospital setting. ${ }^{160,161}$

Studies have also reported resistant $A$. baumannii strains that demonstrated little or no biofilm-producing capacity. Colistin, at sub-inhibitory concentration reduces biofilm formation on urinary catheter surfaces. ${ }^{31}$ It was shown that isolates pretreated with colistin had their adhesion ability reduced significantly and the cultures treated with $0.5 \times$ MIC showed decreased biofilm formation compared to those treated with $0.25 \times$ MIC. ${ }^{31}$ Clinical colistin-resistant (Cstr) strains demonstrated biofilm formation that was significantly decreased in static and dynamic assays $(P<0.001)$ with lower relative fitness $(P<0.05)$ compared with colistin-sensitive counterparts. ${ }^{162}$ This was attributed to conversion of a stop codon to lysine in lipopolysacharide synthesis protein, LpsB in one Cstr strain while there exist a frameshift mutation in CarO together with loss of a 47,969 bp element containing multiple genes associated with biofilm production in a second resistant strain. ${ }^{162}$ Environmental A. baumannii and Klebsiella pneumoniae strains recovered from a university hospital in Brazil from 2009 to 2014 were investigated. ${ }^{22}$ Ten strains of each species were assessed for their biofilm formation capacity, biofilm biomass, and initial adhesion degree in three independent experiments. Although all A. baumannii strains were able to attach to polystyrene plates, some had a lesser degree of adherence than the control. ${ }^{22}$ None of the isolates were strong biofilm producers and no relationship was observed between multidrug resistance and biofilm production. ${ }^{22}$ It was reported that only one of the three clinical MDR $A$. baumannii strains with diverse resistance mechanisms (OXA-51, IMP-1, and VIM-2 type $\beta$-lactamases) produced strong biofilm compared to the control. ${ }^{199}$ The relationship between antibiotic resistance, biofilm formation, and biofilm-specific resistance of 272 clinical isolates of $A$. baumannii was investigated. ${ }^{163}$ Of these, 249 isolates exhibited biofilm formation, of which 63 were stronger biofilm-formers than the A. baumannii strain type ATCC $19606 .{ }^{164}$ The majority of the isolates that formed biofilms were non-MDR, while isolates with high levels of resistance were weak biofilm-formers. ${ }^{164}$ These findings raise questions regarding the mechanisms by which bacteria maintain a balance between biofilm-forming capacity and antibiotic resistance, as well as how resistant strains achieve high levels of biofilm-specific resistance despite producing weak biofilms. ${ }^{164}$

\section{Resistance determinants that contribute to biofilm formation}

Antibiotic resistance is associated with resistance determinants that could be either intrinsic such as the chromosomally-encoded beta-lactamases, drug efflux system, and porins or acquired through insertion elements and integrons. ${ }^{164}$ Some of the resistance determinants identified for A. baumannii include AmpC, VIM, and OXA-10 beta-lactamases, efflux pumps and aminoglycoside-modifying enzymes. ${ }^{165} \mathrm{~A}$. baumannii resistance is also linked to the capacity of the bacteria to produce biofilms. ${ }^{163}$

\section{Beta-lactamases}

The ability of $A$. baumannii to form strong biofilm has been associated with class-D OXA carbapenemase genes. ${ }^{166,167} \mathrm{~A}$ study showed that clinical isolates were able to form stronger biofilm than environmental isolates. ${ }^{152}$ Statistical analysis revealed a significant correlation between the frequency of MDR isolates and biofilm-forming ability $(P=0.008)$. The correlation of biofilm formation and the frequency of biofilm-related genes in clinical and environmental samples revealed a significant association between the frequency of biofilm-associated genes such as blaOXA-51 and the capacity for biofilm formation $(P=0.008) .{ }^{152} \mathrm{PCR}$ analysis of biofilmrelated genes revealed that some genes such as blaOXA-51, blaOXA-23, blaOXA-24, blaOXA-58, blaPER-1, bap, and ompA could be amplified from isolates with the frequencies of ompA, blaPER-1, and bap in clinical and environmental isolates being $100 \%, 53.3 \%, 82.7 \%$, and $100 \%, 37.5 \%$, and $84.4 \%$, respectively. ${ }^{152}$ It was observed that $A$. baumannii containing both blaOXA-23 and bla-OXA-24/40-like genes showed greater initial attachment to microtiter plates and biofilm-forming ability than the imipenem-sensitive 
isolates. ${ }^{163}$ Imipenem-resistant strains were found to harbor blaOXA-24/40-like gene and efficiently attached to microtiter plates, unlike blaOXA-24/40-negative, or imipenemsensitive strains which had weak or no biofilm-forming ability. ${ }^{163}$ The blaOXA-51, blaOXA-23, and blaOXA24/40-like genes could co-exist in strong biofilm-producing, MDR A. baumannii, especially in isolates from hospitalized patients in ICU. ${ }^{163}$ The relationship between biofilm-related genes and antimicrobial resistance of $A$. baumannii samples from 122 patients with lower respiratory tract infections at Fujian Longyan First Hospital, China from January 2013 to September 2014 was examined. ${ }^{96}$ It was observed that the detection rates of $a b a I$ and $c s u E$ were both $59.8 \%$, and those of ompA and blaPER1were $100 \%$ and $0 \%$, respectively. ${ }^{96}$ The study further revealed that resistance to amikacin, ampicillin/sulbactam, and 14 other types of antimicrobials was higher in $a b a I$ - and $c s u E$-positive strains than in abaI- and csuE-negative strains $(P<0.05) .{ }^{96} \mathrm{~A}$. baumannii carrying extended-spectrum beta-lactamase genes formed significantly more biofilms than those without this gene $\left(P<0.005\right.$ and $P<0.001$, respectively).${ }^{96}$ The ability of MDR, clinical isolates of $A$. baumannii to adhere to the epithelial cell surface and to form biofilm in polystyrene plates was reported to be higher in isolates carrying the blaPER-1 gene than those without it. ${ }^{168}$ RT-PCR analysis revealed a positive correlation between the level of expression of the blaPER-1 gene and biofilm formation $(r=0.89 ; P<0.0001)$ and cell adhesiveness $(r=0.74, P<0.006) .{ }^{170} \mathrm{~A}$. baumannii chromosome also encodes an intrinsic blaAmpC and blaOXA-51-like genes that are weakly expressed but do not confer resistance to carbapenems. ${ }^{171}$ However, it was demonstrated that the insertion of an ISAba1 element upstream of the gene conferred carbapenem resistance. ${ }^{171}$ IS elements such as ISAba1, ISAba2, ISAba3, and IS18, have been found to increase the expression of class D beta-lactamase genes (including blaOXA-23-like and blaOXA-58-like genes) when they were inserted immediately upstream due to the presence of an outward-directing promoter at the ends of these IS elements. ${ }^{169,172}$

The revelation that the presence of resistance determinants is one of the key factors that contribute to the ability of $A$. baumannii to produce biofilm, is a serious concern considering the proximity of the isolates within a biofilm and that can facilitate the distribution of resistance genes and plasmids. The ability of a dual-species biofilm to spread blaNDM-1 between both clinical and environmental settings was reported. ${ }^{173}$ The study confirmed that Enterobacte- riaceae strains could transfer blaNDM-1 readily to strong biofilm-producing $P$. aeruginosa and $A$. baumannii in the environment. ${ }^{173}$

\section{Integrons}

A. baumannii has the ability to acquire both narrow- and extended-spectrum beta-lactamases as well as the capacity to acquire integrons, transposons, and insertion elements which can contribute widely to antibiotic resistance. ${ }^{174}$ Some of these resistance determinants have also been associated with the capacity of $A$. baumannii to produce biofilm. For instance, it was revealed that intIl gene mRNA was $\sim 4$ times higher in biofilm cells than that in the planktonic cultured cells and would therefore be much easier for bacteria under biofilm conditions to capture gene cassettes than planktonic cells. ${ }^{175}$ Class I integrons are highly distributed among A. baumannii strains and antibiotic resistance is strongly associated with intII gene cassette-positive strains compared to that of gene cassette-negative strains. ${ }^{175}$ It was reported that MDR A. baumannii may harbour integrons that may differ in different strains. For instance, class 1 and 2 integrons dominate in A. baumannii clonal complex 113/79 (CC109/1 and CC113/79 respectively) isolated from Latin America. ${ }^{176}$ Although these determinants are associated with MDR A. baumannii, their relationship with biofilm-forming ability requires further investigation.

\section{Impact of clonality on A. baumannii biofilm formation}

Associations between biofilm formation and specific clonal lineage were reported. ${ }^{48,177}$ In a study to investigate the factors that contribute to the epidemicity of carbapenemresistant $A$. baumannii, biofilm formation, expression of biofilm-associated genes, and resistance of different epidemic clones: major, minor, and sporadic were compared. The study revealed that the major epidemic clone produced stronger biofilm than the minor and sporadic ones. ${ }^{178}$ Biofilm-associated genes such as abaI, bap, pgaABCD, and $c s u \mathrm{~A} / B A B C D E$ were highly expressed in the major and minor epidemic clones compared to the sporadic clones in sessile conditions. However, the reverse was true in the planktonic state where the minor clone expressed more biofilm-associated genes than the major epidemic clone. The major epidemic clone expressed more $\operatorname{csu} C, \operatorname{csuD}$, and $\operatorname{csu} E$ genes than the minor epidemic clone. ${ }^{178}$ This may account for the epidemicity of CRAB ST191 clone in the hospital environment. Biofilm formation by SMAL, a cluster of a specific clone within MDR 
A. baumannii strains from an Italian hospital showed that the capacity of $A$. baumannii to form biofilm, represented as the ability to adhere to polypropylene surface, was influenced by the growth medium. ${ }^{48}$ Biofilm-production was favored in glucose-based medium and increased the intracellular iron concentration than in rich medium and decreased iron concentration..$^{48}$ A study identified ST2 (international clone II), ST25, and ST78 which produced more biofilm than other sequence types (STs). ${ }^{180}$ In the report that aimed to compare virulence-related traits that dominate in the international clonal lineages of I-III and the emerging genotypes ST25 and ST78, not only did ST78 invade A549 cells more than others, but it also produced more biofilm. ${ }^{180}$ In another study to test if biofilm formation is a major contributor to the success of the international clone 2 (IC2) in infection, it was found that IC2 strains produced less biofilm than other isolates.$^{84}$ This study revealed that biofilm formation may not be the major reason for the great success of a particular clone. ${ }^{84}$ For instance, $A$. baumannii Bap is polymorphic and can be differentiated in three main clonal types both in the repetitive and the $\mathrm{COOH}$ region. ${ }^{55} \mathrm{~A}$ survey of $541 \mathrm{~A}$. baumannii sequenced strains that belong to 108 STs revealed that $29 \%$ featured type- 1 bap, $40 \%$ type- 2 bap, 11\% type-3 bap, while $20 \%$ lacked bap..$^{55}$ It was observed that different $A$. baumannii strains showed high variability in biofilm formation. ${ }^{181}$ Although no relationship existed among carbapenem resistance, isolation site, disease severity of the clinical strains and biofilm production, an increase in biofilm formation was detected in A. baumannii isolates cultured from the same patient upon prolonged hospitalization. ${ }^{181}$ In another study that aimed to investigate the relationship between antibiotic resistance, biofilm formation, and biofilm-specific resistance genes of 272 clinical isolates of $A$. baumannii from a Chinese hospital, pulsed field gel electrophoresis typing yielded 167 different patterns and 103 clusters with a similarity of $80 \%{ }^{164}$ The isolates with the strongest biofilm-producing ability belonged to cluster 9 and 34 with four of the isolates demonstrating the strongest biofilm-producing ability. ${ }^{164}$

It was suggested that biofilm formation is a heterogeneous property among $A$. baumannii, and is not associated with certain clonal types. ${ }^{182} \mathrm{~A}$ study to link a particular clonal lineage to carbapenem resistance and biofilm formation with A. baumannii isolated from Southern Croatia, revealed that no significant difference exists between them. ${ }^{177}$ However, the study showed that higher biofilm producers were isolated from ICUs and respiratory tract infections while lower biofilm producers were isolated from other sources. ${ }^{177}$ The genotype relatedness, antibiotic resistance, and biofilm formation of
A. baumannii isolated from a Portuguese hospital showed that no relationship existed between biofilm formation and pulse type ${ }^{23}$ However, the study showed that urine isolates demonstrated a higher biofilm-forming capacity than those from other sources. ${ }^{23}$

\section{Genomics and proteomics}

Understanding the factors responsible for biofilm formation has been improved by genomic and proteomic studies conducted on genes that are differentially regulated during biofilm formation. Complete genome sequence analysis of a high biofilm-producing A. baumannii strain 1656-2 provided insight into the molecular basis of biofilm formation. ${ }^{183}$ With a genome size of $3,940,614 \mathrm{bp}$, the strain had a guanine-cytosine content of $39.2 \%$, a coding region of $88.0 \%$ with 3,715 open reading frames, 18 predicted rRNA genes, and 71 predicted tRNA genes. ${ }^{183}$ The draft genome sequence of a hyper biofilm-producing A. baumannii strain MAR002 (GEIH/REIPI-Ab2010 Spain) was reported to have an approximate length of 3,717,929 bp and 3,300 protein-coding sequences, with a CG content of $39.09 \% .{ }^{138}$ The pan-genome of 30 completed $A$. baumannii genome sequences was studied and it was reported that they share both intra- and inter-genomic similarities and evolutionary relationships. ${ }^{184}$ The genes responsible for pilus assembly protein, pili assembly chaperone, AdeK, PonA, OmpA, as well as other genes associated with biofilm formation were found to be conserved. ${ }^{184}$ Genes responsible for oxidative stress resistance and alkyl hydroperoxide reductase (AhpC) were up-regulated during biofilm mode compared to the planktonic cells at 48 hours, whereas the AhpC was higher in the planktonic than the biofilm mode at 24 hours. ${ }^{179}$ The AhpC mutant showed a severe growth defect in rich media, promoted early biofilm formation through increased production of EPS, and increased the endogenous hydrogen peroxide production unlike that observed in the minimal media. ${ }^{179}$

A. baumannii 1656-2, a high biofilm-producing clinical isolate was used to determine which proteins are differentially regulated in biofilm cells as compared to planktonic conditions using two-dimensional gel electrophoresis. ${ }^{147}$ Protein identification using MALDI-ToF mass spectrometry revealed that: 1) proteins involved in the process of environmental responses such as Omp, iron transporters, diguanylate cyclase, and sensor histidine kinase/response regulator (PAS-GGEDF-EAL domain); 2) metabolic proteins such as NAD-linked malate dehydrogenase, putative GalE, ProFAR isomerase, as well as nucleoside-diphosphate sugar epimerase, and $\mathrm{N}$-acetylmuramoyl-l-alanine amidase; 
3) antibiotic resistance determinant-related proteins; and 4) gene repair proteins such as exodeoxyribonuclease III and GidA were differentially expressed. ${ }^{147}$ In another study that investigated the mechanisms associated with the ability of A. baumannii to form biofilm, a comparative proteomics analysis of A. baumannii strain ATCC 17978 was performed at three different conditions: exponential, late stationary and biofilm growth phase by two-dimensional gel electrophoresis, MALDI-ToF, and iTRAQ/SCX-LC-MS/MS mass spectrometry analysis. The results were compared with the alterations in the proteome resulting from exposure to salicylate, a 'biofilm-inhibitory compound. ${ }^{159}$ Several novel proteins as well as Baps such as CarO, OmpA, OprD-like, DcaP-like, PstS, LysM, and Omp33, and histidine metabolism (like urocanase) were implicated in biofilm formation. ${ }^{148}$ When MDR and drug-sensitive strains of $A$. baumannii were compared to determine the protein expression levels in A. baumannii resistant phenotype, it was revealed that 114 of the 1,484 proteins present were 2-fold to 66-fold more abundant in the drug-resistant strain, and 99 were 2-fold to 50 -fold less abundant. ${ }^{185}$ The proteins that were over-expressed included porins, drug- and metal-resistant as well as stress- and biofilm-related proteins. ${ }^{185}$

The gene expression pattern of $A$. baumannii ATCC 17978 in the biofilm phenotype was different from that in planktonic cells. ${ }^{186}$ The total mRNA from the planktonic (both exponential and stationary phase cultures) and sessile (biofilm) cultures were obtained, six libraries were prepared using mRNA-Seq protocols from Illumina, and reads were obtained in a HiScanSQ platform and mapped against the complete genome. ${ }^{186}$ Assessing the mRNA expression profiles in biofilm by RNA-sequencing, 1,621 genes were reported to be over-expressed in the biofilms relative to the stationary phase cells and 55 genes were expressed only in the biofilms. ${ }^{186}$ This could explain important changes in amino acid and fatty acid metabolism, motility, active transport, DNA-methylation, iron acquisition, transcriptional regulation, and QS, among other processes. ${ }^{186}$ Comparison of the proteome of drugresistant A. baumannii DU202 with A. baumannii ATCC 17978 strain revealed 72 genes that encode proteins including $\beta$-lactamases, a multidrug resistance efflux pump, and RND multidrug efflux proteins. ${ }^{187}$ In an in vivo transcriptomic study conducted by injecting a mouse intra-peritoneally with A. baumannii ATCC 17978 and assessing the transcriptome after 8 hours with an RNA-Seq with an Illumina platform, 557 genes were found to be down-regulated while 329 genes were up-regulated. ${ }^{188}$ According to the study, up-regulated genes included siderophore iron uptake clusters, genes related to chaperone and protein turnover, while all genes related to types I and IV pili, QS components, and proteins involved in biofilm formation showed reduced expression. ${ }^{188}$

The global metabolite profiling of both planktonic and biofilm forms of the same strain, A. baumannii 1656-2 was compared, using a high-resolution nuclear magnetic resonance spectroscopy and multivariate statistical analysis to investigate the metabolic patterns leading to biofilm formation. ${ }^{189}$ Principal components analysis and orthogonal partial least-squares discriminant analysis score plots showed a distinct separation between planktonic and biofilm cells. ${ }^{189}$ It was concluded that metabolites such as acetates, pyruvate, succinate, and lysine as well as UDP-glucose, AMP, and glutamate are involved in energy metabolism during biofilm formation. ${ }^{189}$

\section{Treatment options of A. baumannii biofilm-associated infections Current and prospective treatments}

The current treatment options available for MDR A. baumannii are suggested for prescribers based on best guess or definitive measures. ${ }^{190} \mathrm{~A}$ joint working meeting consisting of the British Society for Antimicrobial Chemotherapy, Healthcare Infection Society, and the British Infection Association recommended ampicillin/sulbactam for carbapenem-resistant but sulbactam-susceptible A. baumannii infections. ${ }^{190}$ Ceftazidime/avibactam has been reported to have potent activity against Gram-negative bacteria isolated from ICUs including MDR Acinetobacter spp. ${ }^{191}$ The synergistic activity of cefoperazone-sulbactam combined with tigecycline showed good activity against MDR A. baumannii in in vitro studies and could therefore offer a good treatment regime in the future. ${ }^{192}$ Relebactam in combination with imipenem/ cilastatin appeared to be a prospective treatment having currently being used in a clinical trial against imipenemresistant strains. ${ }^{193}$

The treatment of biofilm-associated A. baumannii infections may present a greater challenge due to the chronic nature of such infections. For instance, in a study that aimed to determine the antimicrobial activity of chlorhexidine against bacteria and fungi biofilms, it was observed that although chlorhexidine demonstrated excellent antimicrobial activity for most microorganisms tested in their free form, it was less effective against biofilms of A. baumannii, Escherichia coli, methicillin-resistant Staphylococcus aureus, and P. aeruginosa ${ }^{194}$ Antibiotics and metal resistance profiles of clinical isolates of Acinetobacter haemolyticus in planktonic and biofilm forms revealed a 4-256-fold increase in antibiotic 
and metal concentration, respectively, in order to inhibit biofilm formation over a period of 72 hours compared to the susceptible planktonic counterparts. ${ }^{195}$

Although biofilm formation enhances resistance to antibiotics, combination therapy may be effective to treat biofilmassociated infections. The in vitro stability and efficacy of colistin, tigecycline, and levofloxacin alone or in combination with clarithromycin and/or heparin as lock solutions against biofilm-embedded A. baumannii strains was assessed. ${ }^{196}$ It was reported that catheter lock solution mixed with colistin is effective for treating biofilm-producing A. baumannii associated with catheter infections, while their combination with clarithromycin showed bactericidal activity against the biofilm-embedded A. baumannii strain. ${ }^{196}$ Each antibiotic at 400 times the MIC combined with clarithromycin (200 mg/ $\mathrm{mL})$ and/or heparin $(1,000 \mathrm{U} / \mathrm{mL})$ was compatible. ${ }^{196}$ When compared with other antibiotics alone, the lock solution consisting of colistin was the best agent to eradicate $A$. baumannii embedded in the catheter model. ${ }^{196} \mathrm{~A}$ comparative in vitro efficacy study of colistin-levofloxacin-, colistin-tigecycline-, and tigecycline-levofloxacin-based catheter lock solutions to treat biofilm-associated A. baumannii infections was conducted. Although each antibiotic combination tested at $400 \times$ MIC clearly demonstrated bactericidal activity, colistinlevofloxacin had the most potent antibacterial activity against biofilm-associated A. baumannii catheter infections. ${ }^{197}$ The antimicrobial activity of imipenem and rifampicin alone and in combination against clinical isolates of $A$. baumannii grown in planktonic and biofilm culture was investigated. ${ }^{198}$ The study revealed that imipenem combined with rifampicin demonstrated great capacity and promise for use as a combinatorial therapy for infectious diseases caused by $A$. baumannii. ${ }^{198}$ Hence, there is a need to analyze the relationship of imipenem and rifampicin combination therapy and to also study the effect of this combination therapy on each component of the biofilm, such as PNAG or bacteria in the biofilms using increased clinical isolates. ${ }^{198}$ It was observed that none of the antibiotic regimens of imipenem, colistin, and rifampicin used alone reduced biofilm formation at the MIC of each of the antimicrobial agents, and A. baumannii biofilms were not susceptible to imipenem, colistin or rifampicin as the sole treatment regimen. ${ }^{199}$ However, at 48 hours after incubation, tigecycline $(0.76 \pm 0.23)$, imipenem-rifampicin (1.07 \pm 0.31$)$, and colistin-rifampicin $(1.47 \pm 0.54)$ showed a significant inhibition of biofilm formation compared to the positive controls $(P<0.01) .{ }^{199}$ Meropenem plus sulbactam significantly decreased biofilm biomass and mean thickness, and increased the roughness coefficient of $A$. baumannii bio- film. ${ }^{200}$ However, sulbactam plus tigecycline decreased only the maximum and mean biofilm thickness and not biofilm mass when compared with these antibiotics used alone. ${ }^{200}$ According to the report, these combinations suggested good alternative treatment regimens for carbapenem-resistant A. baumannii and carbapenem-susceptible $A$. baumannii biofilms. ${ }^{200}$

\section{Alternative treatments Quorum quenching}

QS in A. baumannii has been linked to the expression of important proteins such as ppGpp synthase and histidine kinase sensors. ${ }^{125}$ Using proteomic analysis of a rifampicin-resistant variant Acinetobacter sp. strain DR1, it was observed that an AHL lactonase responsible for degrading QS signals was highly up-regulated in both the DR1 and an aqsI mutant, strain causing reduced biofilm formation in the aqsI mutant. ${ }^{201}$ This report represents the first study on the critical role of QS in hexadecane degradation and biofilm formation. ${ }^{201} \mathrm{~A}$ recombinant lactonase was demonstrated to successfully disrupt biofilm formation of clinical isolates of A. baumannii. ${ }^{202}$ This was the first demonstration of the use of recombinant quorum quenching enzymes in the disruption of biofilm formation by the human bacterial pathogen, $A$. baumannii. ${ }^{202}$ The recombinant quorum quenching enzyme, MomL, an enzyme that degrades several QS molecules, reduced biofilm formation while increasing the susceptibility of $P$. aeruginosa PAO1 and A. baumannii LMG 10531 biofilm to different antibiotics in microtiter plates in vitro. ${ }^{203}$ However, MomL was ineffective in treating mixed species and wound-associated biofilm infections. ${ }^{203}$

\section{Natural and organic products}

Natural products such as microbial, plant, and animal derivatives have been demonstrated to be effective to treat A. baumannii infections. Bacterial secondary metabolites have been demonstrated to be effective against $A$. baumannii biofilm production. ${ }^{30}$ The effects of metabolites produced by bacteria isolated from drinking water on the biofilms of A. calcoaceticus, Burkholderia cepacia, Methylobacterium sp., Mycobacterium mucogenicum, Sphingomonas capsulata, and Staphylococcus sp were studied. ${ }^{82}$ It was found that $A$. calcoaceticus, B. cepacia, Methylobacterium sp., and M. mucogenicum biofilms were strongly inhibited by crude cell-free supernatants from the other bacteria. ${ }^{81}$ The isolation and characterization of a new lytic $A$. baumannii bacteriophage that was effective on biofilm were reported. ${ }^{204}$ The phage could 
inhibit $A$. baumannii biofilm formation and also disrupt pre-formed biofilm. ${ }^{205}$

The effect of the antimicrobial peptide (AMP) LL-37 on the integrity of $A$. baumannii biofilm revealed that LL-37 caused structural damage of the biofilm at a low concentration of $2.5 \mu \mathrm{g} / \mathrm{mL} .{ }^{206}$ A natural AMP complex produced by the blowfly maggot Calliphora vicina exhibited strong cell killing and matrix-destroying activity against human pathogenic, antibiotic resistant E. coli, S. aureus, and A. baumannii biofilms, and it was non-toxic to human immune cells. ${ }^{207}$ The complex is reported to contain AMPs from defensin, cecropin, diptericin, and proline-rich peptide families which were likely expressed as a result of bacterial infection. ${ }^{207}$ The anti-biofilm, antimicrobial, and antioxidant activities of the Salvia glutinosa essential oil were effective against MDR A. baumannii with MIC and minimal bactericidal concentration values between $1.25-2.5 \mu \mathrm{L} / \mathrm{mL}$ and $5-10 \mu \mathrm{L} / \mathrm{mL}$, respectively. ${ }^{208}$ A 2-aminoimidazole-based anti-biofilm agent, one of the constituents of a polymeric formulation, was effective against $A$. baumannii biofilm formation and offered no leaching of the 2-aminoimidazole derivative. It was not hemolytic and proved effective in reducing biofilm on indwelling medical devices. ${ }^{209}$

\section{Inorganic substances}

In order to determine the effectiveness of hydrogen peroxide and hydrogen peroxide-based formulations for the eradication of biofilms of three different Gram-negative pathogens associated with MDR phenotypes, it was observed that planktonic cultures and single-species 24-hour biofilms of seven strains of Acinetobacter spp., including clinical isolates, were reduced at the working concentrations of hydrogen peroxide and hydrogen peroxide-based formulations for 1 minute to 24 hours. ${ }^{204}$ However, it was demonstrated that the mixed-culture biofilm cells were more resistant to removal and killing by some biocides, such as hydrogen peroxide and sulfathiazole, than the single-species Acinetobacter biofilm cells. ${ }^{210}$ Oxidizing biocides, such as sodium hypochlorite and hydrogen peroxide, demonstrated a higher potential for biofilm removal and killing than non-oxidizing biocides (sulfathiazole and glutaraldehyde). ${ }^{211}$

Cyclic di-GMP is a well-known and highly conserved second-messenger signal transduction that plays a role in biofilm formation. In silico pharmacophore-based screening identified small-molecule inhibitors of DGC enzymes that synthesize c-di-GMP. ${ }^{212}$ Four small molecules, LP 3134 , LP 3145, LP 4010, and LP 1062 act against c-di-GMP and inhibit biofilm formation in P. aeruginosa and A. bauman- nii in a continuous-flow system and with no toxic effect on eukaryotic cells. ${ }^{212}$ Although the compounds identified from structure-activity relationship studies of the anti-biofilm compound, LP3134, demonstrated an anti-biofilm effect on a silicon catheter, they had no effect on the planktonic growth of A. baumannii. ${ }^{212}$ 5-episinuleptolide, isolated from Sinularia leptoclados, demonstrated a biofilm-inhibitory effect on A. baumannii ATCC 19606 and three MDR A. baumannii strains. ${ }^{213}$ The mechanism of biofilm inhibition was shown to correlate with decreased expression of the pgaABCD locus, which encodes the extracellular polysaccharide, polyPNAG. ${ }^{213}$ It was observed that optimized $N^{2}, N^{4}$-disubstituted quinazoline-2,4-diamines, a dihydrofolate reductase inhibitor, reduced up to $90 \%$ of cells within a biofilm at or near MICs. ${ }^{214}$ The molecule, which has also demonstrated potency in vitro and in vivo against methicillin-resistant $S$. aureus strains, showed great activity against $A$. baumannii and its biofilm at very low MIC when the 6-position was replaced with a halide or an alkyl substituent. ${ }^{214}$ Using a murine model, it was reported that $N^{2}, N^{4}$-disubstituted quinazoline-2,4-diamines proved more effective than tigecycline. ${ }^{214}$ Gallium nitrate $(16 \mu \mathrm{M})$ drastically reduced $A$. baumannii growth and biofilm formation in human serum, whereas $64 \mu \mathrm{M}$ caused massive disruption of the preformed A. baumannii biofilm. ${ }^{210}$ This may be associated with the limiting iron concentration in human serum as iron concentration influences $A$. baumannii biofilm formation in a strain-dependent manner. The effect of silver nanoparticles alone and in combination with biocides and imipenem against planktonic and biofilms of $A$. baumannii ${ }^{85}$ was studied and it was reported that $29.3 \%$ of isolates were susceptible to silver nanoparticles and the agent could inhibit growth and eradicate biofilms produced by the isolates. ${ }^{85}$ The result demonstrated the antagonistic impact of silver nanoparticles against biofilms. ${ }^{85}$ Virstatin at a concentration of $100 \mathrm{mM}$ led to a $38 \%$ decrease of biofilms formed by A. baumannii ATCC17978 when grown under static mode. ${ }^{228}$ According to the report, the production of biofilms grown under dynamic mode with virstatin was also delayed and reduced. ${ }^{215}$

\section{A. baumannii in the African perspective}

Limited to no data are available in scientific literature on biofilm formation of Acinetobacter baumannii isolated from South Africa. This review serves as the first comprehensive and updated information on the physicochemical parameters that affect Acinetobacter baumannii biofilm formation. However, antimicrobial resistance of $A$. baummanii from Africa has been previously reported. The prevalence of 
carbapenem-resistant $A$. baumannii at Mulago Hospital in Kampala Uganda was investigated. ${ }^{216}$ The genes responsible for carbapenem resistance such as blaOXA-23-like (60\%; 9/15), blaOXA-24-like (7\%; 1/15), blaOXA-58-like (13\%; $2 / 15)$, and blaVIM-like $(13 \% ; 2 / 15)$ as well as the class 1 integron in carbapenem-resistant $A$. baumannii were identified. ${ }^{217}$

A variant of NDM-1 in a clinical isolate of $A$. baumanni that had a $\mathrm{C}$ to $\mathrm{G}$ substitution at position 82 resulting in an amino acid substitution of proline to alanine at position 28 was also reported. ${ }^{230}$ Multilocus sequence typing revealed that the isolate haboured a gene belonging to a new sequence type, ST103, and this variant was designated NDM-2. ${ }^{218}$ The prevalence of different Ambler class $\beta$-lactamase-encoding genes such as blaTEM, blaSHV, blaCTX-M, blaVEB, blaPER, blaGES, blaVIM, blaIMP, blaSIM, blaSPM, blaGIM, blaNDM, blaADC, blaOXA-23, blaOXA-24, blaOXA-51, and blaOXA-58, ISAba1, and int 1 among 40 carbapenemresistant $A$. baumannii in Egypt were studied. ${ }^{219}$ blaADC- and blaOXA-51-like genes were present in all the isolates while SHV, CTX-M, VEB, KPC, and MBL encoding genes were absent. blaOXA-23 (50\%), blaOXA-24 (7.5\%), blaTEM(87.5\%), blaPER- (55\%), and blaGES-like (27.5\%) genes were also detected among the isolates. ${ }^{219}$ The isolates were resistant to amoxicillin-clavulanate, aztreonam, cefepime, cefotaxime, and ceftazidime. ${ }^{219} 16 \mathrm{~S}$ rRNA methylase armA was observed together with blaNDM-1 and blaOXA-23 in A. baumannii isolated from tertiary care hospital in Egypt. ${ }^{220}$ The emergence and spread of ST236 and a carbapenemase that resides in clones belonging to ST884, ST945, and ST1096 in Saudi Arabia and Egypt was reported. From Egypt and Saudi Arabia respectively, it was reported that ST236 (CC104), ST208 (CC92), and ST884 were widespread. ${ }^{231}$ Resistance genes detected were ISAba1 (19\%), blaOXA-23 (65\%), blaOXA-24-like (9\%), and blaNDM-1 genes. ${ }^{221}$

The activity of tigecycline against carbapenem-resistant A. baumannii was studied using Vitek-2 colorimetric compact system with Advanced Expert System and modified Hodge test for susceptibility testing and carbapenemase production testing, respectively. ${ }^{216}$ The study revealed that out of the 232 carbapenem-resistant clinical strains, 169 (75.8\%) were susceptible to tigecycline, 37 (16.6\%) were intermediately resistant, and 17 (7.6\%) were resistant. ${ }^{216} A$. baumannii isolates were susceptible to colistin and none of the carbapenem-resistant isolates were susceptible to ampicillin, amoxicillin/clavulanic acid, piperacillin/tazobactam, cefuroxime, cefoxitin, cefepime and nitrofurantoin. ${ }^{216}$ High prevalence of oxacillinases in clinical MDR A. baumannii from Tshwane region, in South Africa was reported. Another report indicated that South African clinical A. baumannii isolates were $100 \%$ resistant to ampicillin, amoxicillin, cefuroxime, cefotaxime, and nitrofurantoin. ${ }^{222}$ Although the isolates showed $67 \%$ or higher resistance rates to ciprofloxacin, imipenem, meropenem, ceftazidime, and cefepime, none were resistant to colistin. ${ }^{222}$ Surprisingly, none of the isolates harbored GES, KPC, NDM, VIM, SPM SIM, PER, and IMP genes and only carried OXA-51 and OXA-23 genes.

\section{Conclusion}

A. baumannii is the first in the critical priority list of pathogens for new antibiotics according to the World Health organization. ${ }^{223,224}$ The extent and levels of MDR A. baumannii in hospitals and the influence of antibiotic resistance on biofilm phenotype is worrisome. Biofilms are estimated to be 1000 times more resistant to antibiotics than the free-living cells. ${ }^{225-227}$ Biofilm formation has been explained to be triggered by the interplay between bacterial cells and environmental factors. A. baumannii is now known to resist almost all currently available antibiotics making it a "superbug" in the hospital setting, specifically in ICUs. There is an urgent need to comprehensively understand the processes and causes of the biofilm phenotype in bacterial cells including A. baumannii, in order to develop antibiofilmspecific drugs. Some of the resistance gene determinants are associated with biofilm formation and serve as a fitness cost in a strain-dependent manner, making it difficult to establish a consensus on the relationships that exist between biofilm formation and environmental triggers, antibiotic resistance, virulence mechanisms as well as the contributions of the source of the bacterial isolates. Hence, future studies should evaluate the influence of bacterial source, environmental factors, antibiotic resistance, and virulence determinants on biofilm formation.

\section{Acknowledgments}

This work was supported by the College of Health Sciences, University of KwaZulu-Natal and the South African National Research Foundation.

\section{Disclosure}

The authors report no conflicts of interest in this work.

\section{References}

1. Evans B, Hamouda A, Amyes S. The rise of carbapenem-resistant Acinetobacter baumannii. Curr. Pharm. Des. 2013;19(2):223-238.

2. Tower K. The genus Acinetobacter. In: Dworkin M, Falkow S, Rosenberg E, Scheleifer KH, Stackebrandt E (editors) The Prokaryotes. New York: Springer; 2006:746-758.

3. Chan JZ, Halachev MR, Loman NJ, Constantinidou C, Pallen MJ. Defining bacterial species in the genomic era: insights from the genus Acinetobacter. BMC Microbiol. 2012;12(1):302. 
4. Munoz-Price LS, Weinstein RA. Acinetobacter infection. N Engl J Med. 2008;358(12):1271-1281.

5. Peleg AY, Jara S, Monga D, Eliopoulos GM, Moellering RC, Mylonakis E. Galleria mellonella as a model system to study Acinetobacter baumannii pathogenesis and therapeutics. Antimicrob Agents Chemother. 2009;53(6):2605-2609.

6. La Scola B, Gundi VA, Khamis A, Raoult D. Sequencing of the rpoB gene and flanking spacers for molecular identification of Acinetobacter species. J Clin Microbiol. 2006;44(3):827-832.

7. Chang HC, Wei YF, Dijkshoorn L, Vaneechoutte M, Tang CT, Chang TC. Species-level identification of isolates of the Acinetobacter calcoaceticus-Acinetobacter baumannii complex by sequence analysis of the 16S-23S rRNA gene spacer region. J Clin Microbiol. 2005;43(4): 1632-1639.

8. Peleg AY, Seifert H, Paterson DL. Acinetobacter baumannii: emergence of a successful pathogen. Clin Microbiol Rev. 2008;21(3): 538-582.

9. Dijkshoorn L, Nemec A, Seifert H. An increasing threat in hospitals: multidrug-resistant Acinetobacter baumannii. Nat Rev Microbiol. 2007;5(12):939-951.

10. Willyard C. Drug-resistant bacteria ranked. Nature. 2017;543(7643):15.

11. Perez F, Hujer AM, Hujer KM, Decker BK, Rather PN, Bonomo RA. Global challenge of multidrug-resistant Acinetobacter baumannii. Antimicrob Agents Chemother. 2007;51(10):3471-3484.

12. Gunn JS, Bakaletz LO, Wozniak DJ. What's on the outside matters: the role of the extracellular polymeric substance of Gram-negative biofilms in evading host immunity and as a target for therapeutic intervention. J Biol Chem. 2016;291(24):12538-12546.

13. Ramos-Gallardo G. Chronic wounds in burn injury: a case report on importance of biofilms. World J Plast Surg. 2016;5(2):175.

14. Sun F, Qu F, Ling Y, et al. Biofilm-associated infections: antibiotic resistance and novel therapeutic strategies. Future Microbiol. 2013;8(7):877-886.

15. Yang J, Toyofuku M, Sakai R, Nomura N. Influence of the alginate production on cell-to-cell communication in Pseudomonas aeruginosa PAO1. Environ Microbiol Rep. 2017;9(3):239-249.

16. Moreno-Gámez S, Sorg RA, Domenech A, et al. Quorum sensing integrates environmental cues, cell density and cell history to control bacterial competence. Nat Commun. 2017;8(1):854.

17. Stewart PS, Costerton JW. Antibiotic resistance of bacteria in biofilms. The Lancet. 2001;358(9276):135-138.

18. Harding CM, Hennon SW, Feldman MF. Uncovering the mechanisms of Acinetobacter baumannii virulence. Nat Rev Microbiol. 2018;16(2):91-102.

19. Abdi-Ali A, Hendiani S, Mohammadi P, Gharavi S. Assessment of biofilm formation and resistance to imipenem and ciprofloxacin among clinical isolates of Acinetobacter baumannii in Tehran. Jundishapur J Microbiol. 2014;7(1).

20. Azizi O, Shahcheraghi F, Salimizand H, et al. Molecular analysis and expression of bap gene in biofilm-forming multi drug-resistant Acinetobacter baumannii. Rep Biochem Mol Bio. 2016;5(1):62.

21. Babapour E, Haddadi A, Mirnejad R, Angaji S-A, Amirmozafari N. Biofilm formation in clinical isolates of nosocomial Acinetobacter baumannii and its relationship with multidrug resistance. Asian Pac J Trop Biomed. 2016;6(6):528-533.

22. de Campos PA, Royer S, da Fonseca Batistão DW, et al. Multidrug resistance related to biofilm formation in Acinetobacter baumannii and Klebsiella pneumonia clinical strains from different pulsotype. Curr Microbiol. 2016;72(5):617-627.

23. Duarte A, Ferreira S, Almeida S, Domingues FC. Clinical isolates of Acinetobacter baumannii from a Portuguese hospital: PFGE characterization, antibiotic susceptibility and biofilm-forming ability. Comp Immunol Microbiol Infect Dis. 2016;45:29-33.

24. Longo F, Vuotto C, Donelli G. Biofilm formation in Acinetobacter baumannii. New Microbiol. 2014;37(2):119-127.
25. Di Domenico EG, Farulla I, Prignano G, et al. Biofilm is a major virulence determinant in bacteria colonization of chronic skin ulcers independently from the multidrug resistant phenotype. Int J Mol Med Sci. 2017;18(5):1077.

26. Oh MH, Choi CH. Role of LuxIR Homologue AnoIR in Acinetobacter nosocomialis and the Effect of Virstatin on the Expression of anoR Gene. J Microbiol Biotechnol. 2015;25(8):1390-1400.

27. Maravić A, Skočibušić M, Fredotović Ž, et al. Urban riverine environment is a source of multidrug-resistant and ESBL-producing clinically important Acinetobacter spp. Environ Sci Pollut Res. 2016;23(4):3525-3535.

28. Mottola C, Mendes JJ, Cristino JM, Cavaco-Silva P, Tavares L, Oliveira M. Polymicrobial biofilms by diabetic foot clinical isolates. Folia Microbiol. 2016;61(1):35-43.

29. Vinod Kumar K, Lall C, Raj RV, Vedhagiri K, Vijayachari P. Coexistence and survival of pathogenic leptospires by formation of biofilm with Azospirillum. FEMS Microbiol Ecol. 2015;91(6).

30. Seo H, Kim J, Jung J, Jin HM, Jeon CO, Park W. Complexity of cellcell interactions between Pseudomonas sp. AS1 and Acinetobacter oleivorans DR1: metabolic commensalism, biofilm formation and quorum quenching. Res Microbiol. 2012;163(3):173-181.

31. Pour NK, Dusane DH, Dhakephalkar PK, Zamin FR, Zinjarde SS, Chopade BA. Biofilm formation by Acinetobacter baumannii strains isolated from urinary tract infection and urinary catheters. FEMS Immunol Med Microbiol. 2011;62(3):328-338.

32. Renner LD, Weibel DB. Physicochemical regulation of biofilm formation. MRS Bull. 2011;36(5):347-355.

33. Gentile V, Frangipani E, Bonchi C, Minandri F, Runci F, Visca P. Iron and Acinetobacter baumannii biofilm formation. Pathogens. 2014;3(3):704-719.

34. Bae YM, Zheng L, Hyun JE, Jung KS, Heu S, Lee SY. Growth characteristics and biofilm formation of various spoilage bacteria isolated from fresh produce. J Food Sci. 2014;79(10).

35. Di Ciccio P, Vergara A, Festino A, et al. Biofilm formation by Staphylococcus aureus on food contact surfaces: relationship with temperature and cell surface hydrophobicity. Food Control. 2015;50:930-936.

36. Han N, Mizan MFR, Jahid IK, Ha S-D. Biofilm formation by Vibrio parahaemolyticus on food and food contact surfaces increases with rise in temperature. Food Control. 2016;70:161-166.

37. Martí S, Rodríguez-Baño J, Catel-Ferreira M, et al. Biofilm formation at the solid-liquid and air-liquid interfaces by Acinetobacter species. BMC Res Notes. 2011;4(1):5.

38. Assaidi A, Ellouali M, Latrache H, et al. Effect of temperature and plumbing materials on biofilm formation by Legionella pneumophila serogroup 1 and 2-15. J Adhes Sci Technol. 2018:1-14.

39. De Silva PM, Chong P, Fernando DM, Westmacott G, Kumar A. Effect of incubation temperature on antibiotic resistance and virulence factors of Acinetobacter baumannii ATCC 17978. Antimicrob Agents Chemother. 2018;62(1):e01514-01517.

40. M'hamedi I, Hassaine H, Bellifa S, Lachachi M, Terki IK, Djeribi R. Biofilm formation by Acinetobacter baumannii isolated from medical devices at the intensive care unit of the University Hospital of Tlemcen (Algeria). Afr J Microbiol Res. 2014;8(3):270-276.

41. Nyenje ME, Green E, Ndip RN. Evaluation of the effect of different growth media and temperature on the suitability of biofilm formation by Enterobacter cloacae strains isolated from food samples in South Africa. Molecules. 2013;18(8):9582-9593.

42. Stepanović S, Ćirković I, Mijač V, Švabić-Vlahović M. Influence of the incubation temperature, atmosphere and dynamic conditions on biofilm formation by Salmonella spp. Food Microbiol. 2003;20(3): 339-343.

43. Bucs SS, Linares RV, Van Loosdrecht M, Kruithof JC, Vrouwenvelder JS. Impact of organic nutrient load on biomass accumulation, feed channel pressure drop increase and permeate flux decline in membrane systems. Water Res. 2014;67:227-242. 
44. Oliveira NM, Martinez-Garcia E, Xavier J, et al. Biofilm formation as a response to ecological competition. PLoS Biol. 2015;13(7):e1002191.

45. Drescher K, Nadell CD, Stone HA, Wingreen NS, Bassler BL. Solutions to the public goods dilemma in bacterial biofilms. Curr Biol. 2014;24(1):50-55.

46. Rinaudi L, Fujishige NA, Hirsch AM, Banchio E, Zorreguieta A, Giordano W. Effects of nutritional and environmental conditions on Sinorhizobium meliloti biofilm formation. Res Microbiol. 2006;157(9):867-875.

47. Assaf D, Steinberg D, Shemesh M. Lactose triggers biofilm formation by Streptococcus mutans. Int Dairy J. 2015;42:51-57.

48. Nucleo E, Steffanoni L, Fugazza G, et al. Growth in glucose-based medium and exposure to subinhibitory concentrations of imipenem induce biofilm formation in a multidrug-resistant clinical isolate of Acinetobacter baumannii. BMC Microbiol. 2009;9(1):270.

49. Leme AP, Koo H, Bellato C, Bedi G, Cury J. The role of sucrose in cariogenic dental biofilm formation-new insight. J Dent Res. 2006;85(10):878-887.

50. Rodriguez A, Autio WR, McLandsborough LA. Effect of surface roughness and stainless steel finish on Listeria monocytogenes attachment and biofilm formation. J Food Prot. 2008;71(1):170-175.

51. Song F, Koo H, Ren D. Effects of material properties on bacterial adhesion and biofilm formation. J Dent Res. 2015;94(8): 1027-1034.

52. Han A, Li X, Huang B, et al. The effect of titanium implant surface modification on the dynamic process of initial microbial adhesion and biofilm formation. Int J Adhes Adhes. 2016;69:125-132.

53. Greene C, Wu J, Rickard AH, Xi C. Evaluation of the ability of Acinetobacter baumannii to form biofilms on six different biomedical relevant surfaces. Lett Appl Microbiol. 2016;63(4):233-239.

54. Orsinger-Jacobsen SJ, Patel SS, Vellozzi EM, et al. Use of a stainless steel washer platform to study Acinetobacter baumannit adhesion and biofilm formation on abiotic surfaces. Microbiology. 2013;159(12):2594-2604.

55. De Gregorio E, Del Franco M, Martinucci M, Roscetto E, Zarrilli R, Di Nocera PP. Biofilm-associated proteins: news from Acinetobacter. BMC Genomics. 2015;16(1):933.

56. Goh HS, Beatson SA, Totsika M, et al. Molecular analysis of the Acinetobacter baumannii biofilm-associated protein. J Appl Environ Microbiol. 2013;79(21):6535-6543.

57. Hayrapetyan H, Siezen R, Abee T, Nierop Groot M. Comparative genomics of iron-transporting systems in Bacillus cereus strains and impact of iron sources on growth and biofilm formation. Front Microbiol. 2016;7:842.

58. Oglesby-Sherrouse AG, Djapgne L, Nguyen AT, Vasil AI, Vasil ML. The complex interplay of iron, biofilm formation, and mucoidy affecting antimicrobial resistance of Pseudomonas aeruginosa. Pathog Dis. 2014;70(3):307-320.

59. Modarresi F, Azizi O, Shakibaie MR, Motamedifar M, Mosadegh E, Mansouri S. Iron limitation enhances acyl homoserine lactone (AHL) production and biofilm formation in clinical isolates of Acinetobacter baumannii. Virulence. 2015;6(2):152-161.

60. Wiens JR, Vasil AI, Schurr MJ, Vasil ML. Iron-regulated expression of alginate production, mucoid phenotype, and biofilm formation by Pseudomonas aeruginosa . MBio. 2014;5(1):e01010-01013.

61. Jones CJ, Ryder CR, Mann EE, Wozniak DJ. AmrZ modulates Pseudomonas aeruginosa biofilm architecture by directly repressing transcription of the psl operon. J Bacteriol. 2013;195(8):1637-1644.

62. Kim HA, Ryu SY, Seo I, Suh S-I, Suh M-H, Baek W-K. Biofilm formation and colistin susceptibility of Acinetobacter baumannii isolated from Korean nosocomial samples. Microb Drug Resist. 2015;21(4):452-457.

63. Minandri F, Imperi F, Frangipani E, et al. Role of iron uptake systems in Pseudomonas aeruginosa virulence and airway infection. Infect Immun. 2016;84(8):2324-2335.
64. Philips J, Rabaey K, Lovley DR, Vargas M. Biofilm formation by Clostridium ljungdahlii is induced by sodium chloride stress: experimental evaluation and transcriptome analysis. PLoS One 2017;12(1):e0170406.

65. Singh S, Singh SK, Chowdhury I, Singh R. Understanding the mechanism of bacterial biofilm resistance to antimicrobial agents. Open Microbiol J. 2017;11:53.

66. Padilla-Chacón D, Castillo-Juárez I, Muñoz-Cazares N, GarcíaContreras R. Gene expression and enhanced antimicrobial resistance in biofilms. Biofilms in Plant and Soil Health. 2017:231.

67. Carrel M, Morales VL, Beltran MA, et al. Biofilms in 3D porous media: Delineating the influence of the pore network geometry, flow and mass transfer on biofilm development. Water Res. 2018;134:280-291.

68. Araújo PA, Malheiro J, Machado I, Mergulhão F, Melo L, Simões M. Influence of flow velocity on the characteristics of Pseudomonas fluorescens biofilms. J Environ Eng. 2016;142(7):04016031.

69. Blanc V, Isabal S, Sanchez M, et al. Characterization and application of a flow system for in vitro multispecies oral biofilm formation. J Periodontal Res. 2014;49(3):323-332.

70. Bhargava N, Sharma P, Capalash N. Quorum sensing in Acinetobacter baumannii. In: Kalia VC (editor) Quorum sensing vs quorum quenching: a battle with no end in sight. New Delhi: Springer; 2015:101-113.

71. Subhadra B, Oh MH, Choi CH. Quorum sensing in Acinetobacter: With special emphasis on antibiotic resistance, biofilm formation and quorum quenching. AIMS Microbiol. 2016;2:27-41.

72. López M, Mayer C, Fernández-García L, et al. Quorum sensing network in clinical strains of $A$. baumannii: AidA is a new quorum quenching enzyme. PloS One. 2017;12(3):e0174454.

73. Harmsen M, Yang L, Pamp SJ, Tolker-Nielsen T. An update on Pseudomonas aeruginosa biofilm formation, tolerance, and dispersal. FEMS Immunol Med Microbiol. 2010;59(3):253-268

74. Hong Y, Brown DG. Electrostatic behavior of the charge-regulated bacterial cell surface. Langmuir. 2008;24(9):5003-5009.

75. Katsikogianni M, Missirlis Y. Interactions of bacteria with specific biomaterial surface chemistries under flow conditions. Acta Biomater. 2010;6(3):1107-1118.

76. Soni KA, Balasubramanian AK, Beskok A, Pillai SD. Zeta potential of selected bacteria in drinking water when dead, starved, or exposed to minimal and rich culture media. Curr Microbiol. 2008;56(1): 93-97.

77. Siboni N, Lidor M, Kramarsky-Winter E, Kushmaro A. Conditioning film and initial biofilm formation on ceramics tiles in the marine environment. FEMS Microbiol Lett. 2007;274(1):24-29.

78. Breitbach AS, Broderick AH, Jewell CM, et al. Surface-mediated release of a synthetic small-molecule modulator of bacterial quorum sensing: Gradual release enhances activity. Chem Commun. 2011;47(1):370-372.

79. Jones EM, Cochrane CA, Percival SL. The effect of $\mathrm{pH}$ on the extracellular matrix and biofilms. Adv Wound Care. 2015;4(7):431-439.

80. da Silva Meira QG, de Medeiros Barbosa I, Athayde AJAA, de Siqueira-Júnior JP, de Souza EL. Influence of temperature and surface kind on biofilm formation by Staphylococcus aureus from food-contact surfaces and sensitivity to sanitizers. Food Control. 2012;25(2):469-475.

81. Simões LC, Simoes M, Vieira MJ. Intergeneric coaggregation among drinking water bacteria: evidence of a role for Acinetobacter calcoaceticus as a bridging bacterium. J Appl Environ Microbiol. 2008;74(4):1259-1263.

82. Bai X, Wu F, Zhou B, Zhi X. Biofilm bacterial communities and abundance in a full-scale drinking water distribution system in Shanghai. J Water Health. 2010;8(3):593-600.

83. Mahapatra A, Padhi N, Mahapatra D, et al. Study of biofilm in bacteria from water pipelines. J Clin Diagn Res. 2015;9(3):DC09.

84. Hu Y, He L, Tao X, Meng F, Zhang J. Biofilm may not be necessary for the epidemic spread of Acinetobacter baumannii. Sci Rep. 2016;6. 
85. Hendiani S, Abdi-Ali A, Mohammadi P, Kharrazi S. Synthesis of silver nanoparticles and its synergistic effects in combination with imipenem and two biocides against biofilm producing Acinetobacter baumannii. Nanomed J. 2015;2(4):291-298.

86. de Breij A, Gaddy J, van der Meer J, et al. CsuA/BABCDE-dependent pili are not involved in the adherence of Acinetobacter baumannii ATCC19606T to human airway epithelial cells and their inflammatory response. Res Microbiol. 2009;160(3):213-218.

88. Kim IH, Wen Y, Son J-S, Lee K-H, Kim K-S. The fur-iron complex modulates expression of the quorum-sensing master regulator, $\mathrm{SmcR}$, to control expression of virulence factors in Vibrio vulnificus. Infect Immun. 2013;81(8):2888-2898.

89. Johnstone TC, Nolan EM. Beyond iron: non-classical biological functions of bacterial siderophores. Dalton Trans. 2015;44(14):6320-6339.

90. Loehfelm TW, Luke NR, Campagnari AA. Identification and characterization of an Acinetobacter baumannii biofilm-associated protein. J Bacteriol. 2008;190(3): 1036-1044.

91. Gaddy JA, Tomaras AP, Actis LA. The Acinetobacter baumannii 19606 OmpA protein plays a role in biofilm formation on abiotic surfaces and in the interaction of this pathogen with eukaryotic cells. Infect Immun. 2009;77(8):3150-3160.

92. Harding CM, Tracy EN, Carruthers MD, Rather PN, Actis LA, Munson RS. Acinetobacter baumannii strain M2 produces type IV pili which play a role in natural transformation and twitching motility but not surface-associated motility. MBio. 2013;4(4):e00360-00313.

93. Harding CM, Hennon SW, Feldman MF. Uncovering the mechanisms of Acinetobacter baumannii virulence. Nat Rev Microbiol. 2018;16(2):91

94. Mussi MA, Relling VM, Limansky AS, Viale AM. CarO, an Acinetobacter baumannii outer membrane protein involved in carbapenem resistance, is essential for 1-ornithine uptake. FEBS Lett. 2007;581(29):5573-5578.

95. Brossard KA, Campagnari AA. The Acinetobacter baumannii biofilmassociated protein plays a role in adherence to human epithelial cells. Infect Immun. 2012;80(1):228-233.

96. Liu H, Wu Y-Q, Chen L-P, et al. Biofilm-related genes: analyses in multi-antibiotic resistant Acinetobacter baumannii isolates from mainland China. Med Sci Monit. 2016;22:1801.

97. Balcázar JL, Subirats J, Borrego CM. The role of biofilms as environmental reservoirs of antibiotic resistance. Front Microbiol. 2015;6.

98. Kor S-B, Chew C-H. Distribution of the multidrug efflux pump genes adeA, adeI, adeJ, adeY and integrons in clinical isolates of Acinetobacter baumannii from Malaysian hospitals. Biomed Res. 2014;25(2).

99. Yoon E-J, Chabane YN, Goussard S, et al. Contribution of resistance-nodulation-cell division efflux systems to antibiotic resistance and biofilm formation in Acinetobacter baumannii. MBio. 2015;6(2):e00309-00315.

100. Zhang T, Wang M, Xie Y, et al. Active efflux pump adeB is involved in multidrug resistance of Acinetobacter baumannii induced by antibacterial agents. Exp Ther Med. 2017;13(4):1538-1546.

101. Richmond GE, Evans LP, Anderson MJ, et al. The Acinetobacter baumannii two-component system AdeRS regulates genes required for multidrug efflux, biofilm formation, and virulence in a strain-specific manner. MBio. 2016;7(2):e00430-00416.

102. Choi AH, Slamti L, Avci FY, Pier GB, Maira-Litrán T. The pgaABCD locus of Acinetobacter baumannii encodes the production of poly$\beta-1-6-\mathrm{N}$-acetylglucosamine, which is critical for biofilm formation. $J$ Bacteriol. 2009;191(19):5953-5963.

103. Wright MS, Jacobs MR, Bonomo RA, Adams MD. Transcriptome Remodeling of Acinetobacter baumannii during infection and treatment. MBio. 2017;8(2):e02193-02116.

104. Smani Y, Fàbrega A, Roca I, Sánchez-Encinales V, Vila J, Pachón J. Role of OmpA in the multidrug resistance phenotype of Acinetobacter baumannii. Antimicrob Agents Chemother. 2014;58(3):1806-1808.

105. Gaddy JA, Actis LA. Regulation of Acinetobacter baumannii biofilm formation. Future Microbiol. 2009;4(3) 273-8.
106. Rumbo C, Gato E, López M, et al. The contribution of efflux pumps, porins and $\beta$-lactamases to multi-drug resistance in clinical isolates of Acinetobacter baumannii. Antimicrob Agents Chemother. 2013;57(11):5247-5257.

107. Chan AP, Sutton G, DePew J, et al. A novel method of consensus panchromosome assembly and large-scale comparative analysis reveal the highly flexible pan-genome of Acinetobacter baumannii. Genome Biol. 2015;16(1):143.

108. Lannan FM, O'conor DK, Broderick JC, et al. Evaluation of virulence gene expression patterns in Acinetobacter baumannii using quantitative real-time polymerase chain reaction array. Mil Med. 2016;181(9):1108-1113.

109. Novovic K, Mihajlovic S, Vasiljevic Z, Filipic B, Begovic J, Jovcic B. Carbapenem-resistant Acinetobacter baumannii from Serbia: Revision of CarO classification. PloS One. 2015;10(3):e0122793.

110. Russo TA, MacDonald U, Beanan JM, et al. Penicillin-binding protein $7 / 8$ contributes to the survival of Acinetobacter baumannii in vitro and in vivo. J Infect Immu. 2009;199(4):513-521.

111. Carruthers MD, Nicholson PA, Tracy EN, Munson Jr RS. Acinetobacter baumannii utilizes a type VI secretion system for bacterial competition. PloS One. 2013;8(3):e59388.

112. Kenyon JJ, Hall RM. Variation in the complex carbohydrate biosynthesis loci of Acinetobacter baumannii genomes. PLoS One. 2013;8(4):e62160

113. Shashkov AS, Kenyon JJ, Senchenkova SyN, et al. Acinetobacter baumannii $\mathrm{K} 27$ and $\mathrm{K} 44$ capsular polysaccharides have the same $\mathrm{K}$ unit but different structures due to the presence of distinct wzy genes in otherwise closely related $\mathrm{K}$ gene clusters. Glycobiology. 2015;26(5):501-508

114. Sigala J-C, Suárez BP, Lara AR, et al. Genomic and physiological characterization of a laboratory-isolated Acinetobacter schindleri ACE strain that quickly and efficiently catabolizes acetate. Microbiology. 2017;163(7):1052-1064.

115. Hasan T, Choi CH, Oh MH. Genes involved in the biosynthesis and transport of acinetobactin in Acinetobacter baumannii. Genomics Inform. 2015;13(1):2-6.

116. Gaddy JA, Arivett BA, McConnell MJ, López-Rojas R, Pachón J, Actis LA. Role of acinetobactin-mediated iron acquisition functions in the interaction of Acinetobacter baumannii strain ATCC 19606T with human lung epithelial cells, Galleria mellonella caterpillars, and mice. Infect Immun. 2012;80(3):1015-1024.

117. Sefidi MD, Rasooli I, Owlia P, Talei D, Astaneh SDA, Nazarian S. Adjuvant role of Pseudomonas flagellin for Acinetobacter baumannii biofilm associated protein. World J Methodol. 2016;6(3):190.

118. Sefid F, Rasooli I, Jahangiri A, Bazmara H. Functional exposed amino acids of BauA as potential immunogen against Acinetobacter baumannii. Acta Biotheor. 2015;63(2):129-149.

119. Liu Y, Liu X. Detection of AmpC $\beta$-lactamases in Acinetobacter baumannii in the Xuzhou region and analysis of drug resistance. Exp Ther Med. 2015;10(3):933-936.

120. Rodríguez-Martínez J-M, Poirel L, Nordmann P. Genetic and functional variability of AmpC-type $\beta$-lactamases from Acinetobacter baumannii. Antimicrob Agents Chemother. 2010;54(11):4930-4933.

121. Tsakris A, Ikonomidis A, Pournaras S, et al. VIM-1 metallo- $\beta$-lactamase in Acinetobacter baumannii. Emerg Infect Dis. 2006;12(6):981.

122. Rodríguez CH, Yarhui NB, Nastro M, et al. Molecular epidemiology of carbapenem-resistant Acinetobacter baumannii in South America. J Med Microbiol. 2016;65(10):1088-1091.

123. Davoodi S, Boroumand MA, Sepehriseresht S, Pourgholi L. Detection of VIM-and IMP-type metallo-beta-lactamase genes in Acinetobacter baumannii isolates from patients in two hospitals in Tehran. Iran $\mathrm{J}$ Biotechnol. 2015;13(1):63-67.

124. Badmasti F, Siadat SD, Bouzari S, Ajdary S, Shahcheraghi F. Molecular detection of genes related to biofilm formation in multidrug-resistant Acinetobacter baumannii isolated from clinical settings. J Med Microbiol. 2015;64(Pt 5):538-543. 
125. Chen H, Cao J, Zhou C, Liu H, Zhang X, Zhou T. Biofilm formation restrained by subinhibitory concentrations of tigecyclin in Acinetobacter baumannii is associated with downregulation of efflux pumps. Chemotherapy. 2017;62(2):128-133.

126. Li X-Z, Nikaido H. Efflux-mediated drug resistance in bacteria. Drugs. 2004;64(2):159-204.

127. Coyne S, Courvalin P, Périchon B. Efflux-mediated antibiotic resistance in Acinetobacter spp. Antimicrob Agents Chemother 2011;55(3):947-953.

128. Damier-Piolle L, Magnet S, Brémont S, Lambert T, Courvalin P. AdeIJK, a resistance-nodulation-cell division pump effluxing multiple antibiotics in Acinetobacter baumannii. Antimicrob Agents Chemother. 2008;52(2):557-562.

129. Krishnamoorthy S, Shah BP, Lee HH, Martinez LR. Microbicides alter the expression and function of RND-type efflux pump AdeABC in biofilm-associated cells of Acinetobacter baumannii clinical isolates. Antimicrob Agents Chemother. 2016;60(1):57-63.

130. He X, Lu F, Yuan F, et al. Biofilm formation caused by clinical Acinetobacter baumannii isolates is associated with overexpression of the AdeFGH efflux pump. Antimicrob Agents Chemother. 2015;59(8):4817-4825.

131. Pokrovskaya V, Poloczek J, Little DJ, Griffiths H, Howell PL, Nitz M. Functional characterization of Staphylococcus epidermidis IcaB, a de-N-acetylase important for biofilm formation. Biochemistry. 2013;52(32):5463-5471.

132. Prashanth K, Vasanth T, Saranathan R, Makki AR, Pagal S. Antibiotic resistance, biofilms and quorum sensing in Acinetobacter species. In: Marina P (editor). Antibiotic Resistant Bacteria-A Continuous Challenge in the New Millennium. Croatia: In Tech; 2012.

133. Xiang J, Sun Z, Yang X, Huan J. Changes in expression of gene aba I in biofilm of Acinetobacter baumannii strains isolated from burn patients. Zhonghua Shao Shang Za Zhi. 2012;28(2):101-105.

134. Wright MS, Iovleva A, Jacobs MR, Bonomo RA, Adams MD. Genome dynamics of multidrug-resistant Acinetobacter baumannii during infection and treatment. Genome Med. 2016;8(1):26.

135. Cao Q, Wang Y, Chen F, et al. A novel signal transduction pathway that modulates rhl quorum sensing and bacterial virulence in Pseudomonas aeruginosa . PLoS Pathog. 2014;10(8):e1004340.

136. Luo LM, Wu LJ, Xiao YL, et al. Enhancing pili assembly and biofilm formation in Acinetobacter baumannii ATCC19606 using non-native acyl-homoserine lactones. BMC Microbiol. 2015;15(1):62.

137. Liou ML, Soo PC, Ling SR, Kuo HY, Tang CY, Chang KC. The sensor kinase BfmS mediates virulence in Acinetobacter baumannii. J Microbiol Immun Infect. 2014;47(4):275-281.

138. Álvarez-Fraga L, López M, Merino M, et al. Draft genome sequence of the biofilm-hyperproducing Acinetobacter baumannii clinical strain MAR002. Genome Announc. 2015;3(4):e00824-00815.

139. Álvarez-Fraga L, Pérez A, Rumbo-Feal S, et al. Analysis of the role of the LH92_11085 gene of a biofilm hyper-producing Acinetobacter baumannii strain on biofilm formation and attachment to eukaryotic cells. Virulence. 2016;7(4):443-455.

140. Bhargava N, Sharma P, Capalash N. N-acyl homoserine lactone mediated interspecies interactions between $A$. baumannii and $P$. aeruginosa. Biofouling. 2012;28(8):813-822.

141. Bhargava N, Sharma P, Capalash N. Quorum sensing in Acinetobacter: an emerging pathogen. Crit Rev Microbiol. 2010;36(4):349-360.

142. Kalia VC. Quorum sensing vs quorum quenching: a battle with no end in sight. Springer, New Delhi, India; 2015.

143. Anbazhagan D, Mansor M, Yan GOS, Yusof MYM, Hassan H, Sekaran SD. Detection of quorum sensing signal molecules and identification of an autoinducer synthase gene among biofilm forming clinical isolates of Acinetobacter spp. PLoS One. 2012;7(7):e36696.

144. Sahu PK, Iyer PS, Barage SH, Sonawane KD, Chopade BA. Characterization of the algC gene expression pattern in the multidrug resistant Acinetobacter baumannii AIIMS 7 and correlation with biofilm development on abiotic surface. Sci World J. 2014; 2014
145. Zarrilli R. Acinetobacter baumannii virulence determinants involved in biofilm growth and adherence to host epithelial cells. Virulence. 2016;7(4):367-368.

146. Choi CH, Lee JS, Lee YC, Park TI, Lee JC. Acinetobacter baumannii invades epithelial cells and outer membrane protein A mediates interactions with epithelial cells. BMC Microbiol. 2008;8(1):216.

147. Shin J-H, Lee H-W, Kim S-M, Kim J. Proteomic analysis of Acinetobacter baumannii in biofilm and planktonic growth mode. J Microbiol. 2009;47(6):728-735.

148. Cabral MP, Soares NC, Aranda J, et al. Proteomic and functional analyses reveal a unique lifestyle for Acinetobacter baumannii biofilms and a key role for histidine metabolism. J Proteome Res. 2011;10(8):3399-3417.

149. Perez A, Merino M, Rumbo-Feal S, et al. The FhaB/FhaC two-partner secretion system is involved in adhesion of Acinetobacter baumannii AbH12O-A2 strain. Virulence. 2017:1-16.

150. Álvarez-Fraga L, Rumbo-Feal S, Pérez A, et al. Global assessment of small RNAs reveals a non-coding transcript involved in biofilm formation and attachment in Acinetobacter baumannii ATCC 17978. PLoS One. 2017;12(8):e0182084.

151. García-Garmendia J-L, Ortiz-Leyba C, Garnacho-Montero J, et al. Risk factors for Acinetobacter baumannii nosocomial bacteremia in critically ill patients: a cohort study. Clin Infect Dis. 2001;33(7):939-946.

152. Bardbari AM, Arabestani MR, Karami M, Keramat F, Alikhani MY, Bagheri KP. Correlation between ability of biofilm formation with their responsible genes and MDR patterns in clinical and environmental Acinetobacter baumannii isolates. Microb Pathog. 2017;108:122-128.

153. Kumari AMS, Routray A, Yadav D, Madhavan R. Imipenem resistance and biofilm production in Acinetobacter. Drug Invent Today. 2013;5(3):256-258.

154. Badave GK, Kulkarni D. Biofilm producing multidrug resistant Acinetobacter baumannii: an emerging challenge. J Clin Diagn Res. 2015;9(1):DC08.

155. Nahar A, Anwar S, Miah MRA. Association of biofilm formation with antimicrobial resistance among the Acinetobacter species in a tertiary care hospital in Bangladesh. J Med. 2013;14(1):28.

156. Bocanegra-Ibarias P, Pena-López C, Camacho-Ortiz A, et al. Genetic characterisation of drug resistance and clonal dynamics of Acinetobacter baumannii in a hospital setting in Mexico. Int J Antimicrob Agents. 2015;45(3):309-313.

157. Huang X, Xiang J, Song F, Huan J. Effects of topical agents for burns on Acinetobacter baumannii within biofilm. Zhonghua Shao Shang Za Zhi. 2012;28(2):106-110.

158. Zhang D, Xia J, Xu Y, et al. Biological features of biofilm-forming ability of Acinetobacter baumannii strains derived from 121 elderly patients with hospital-acquired pneumonia. Clin Exp Med. 2016;16(1):73-80.

159. Han X, Li Q, Shen L, Hu D, Qu Y. Correlation between the biofilmforming ability, biofilm-related genes and antimicrobial resistance of Acinetobacter baumannii. Zhonghua Wei Zhong Bing Ji Jiu Yi Xue. 2014;26(9):639-643.

160. Guo HN, Xiang J. [Influences of abaR gene on biofilm formation of Acinetobacter baumannii]. Zhonghua Shao Shang Za Zhi. 2017;20;33(4):200-205. Chinese.

161. Hall CW, Mah T-F. Molecular mechanisms of biofilm-based antibiotic resistance and tolerance in pathogenic bacteria. FEMS Microbiol Lett Rev. 2017;41(3):276-301.

162. Dafopoulou K, Xavier BB, Hotterbeekx A, et al. Colistin-resistant Acinetobacter baumannii clinical strains with deficient biofilm formation. Antimicrob Agents Chemother 2016;60(3):1892-1895.

163. Azizi O, Shakibaie MR, Modarresi F, Shahcheraghi F. Molecular detection of class-D OXA carbapenemase genes in biofilm and non-biofilm forming clinical isolates of Acinetobacter baumannii. Jundishapur $\mathbf{J}$ Microbiol. 2015;8(1).

164. Qi L, Li H, Zhang C, et al. Relationship between antibiotic resistance, biofilm formation, and biofilm-specific resistance in Acinetobacter baumannii. Front Microbiol. 2016;7. 
165. Lee C-R, Lee JH, Park M, et al. Biology of Acinetobacter baumannii: pathogenesis, antibiotic resistance mechanisms, and prospective treatment options. Front Cell Infect Microbiol. 2017;7:55.

166. Poirel L, Nordmann P. Carbapenem resistance in Acinetobacter baumannii: mechanisms and epidemiology. Clin Microbiol Infect. 2006;12(9):826-836.

167. Héritier C, Poirel L, Lambert T, Nordmann P. Contribution of acquired carbapenem-hydrolyzing oxacillinases to carbapenem resistance in Acinetobacter baumannii. Antimicrob Agents Chemother. 2005;49(8):3198-3202.

168. Lee HW, Koh Y, Kim J, et al. Capacity of multidrug-resistant clinical isolates of Acinetobacter baumannii to form biofilm and adhere to epithelial cell surfaces. Clin Microbiol Infect. 2008;14(1):49-54.

169. Zarrilli R, Vitale D, Di Popolo A, et al. A plasmid-borne blaOXA-58 gene confers imipenem resistance to Acinetobacter baumannii isolates from a Lebanese hospital. Antimicrob Agents Chemother. 2008;52(11):4115-4120.

170. Lee HW, Koh Y, Kim J, et al. Capacity of multidrug-resistant clinical isolates of Acinetobacter baumannii to form biofilm and adhere to epithelial cell surfaces. Clin Microbiol Infect. 2008;14(1):49-54.

171. Turton JF, Ward ME, Woodford N, et al. The role of IS Aba1 in expression of OXA carbapenemase genes in Acinetobacter baumannii. FEMS Microbiol Lett. 2006;258(1):72-77.

172. Hsu L-Y, Apisarnthanarak A, Khan E, Suwantarat N, Ghafur A, Tambyah PA. Carbapenem-resistant Acinetobacter baumannii and Enterobacteriaceae in South and Southeast Asia. Clin Microbiol Rev. 2017;30(1):1-22.

173. Tanner WD, Atkinson RM, Goel RK, et al. Horizontal transfer of the blaNDM-1 gene to Pseudomonas aeruginosa and Acinetobacter baumannii in biofilms. FEMS Microbiol Lett. 2017;364(8).

174. Pagano M, Martins AF, Barth AL. Mobile genetic elements related to carbapenem resistance in Acinetobacter baumannii. Braz J Microbiol. 2016;47(4):785-792

175. Li L, Xia Z, Hu Z, Zhou Z, Li H. Expression of Class I integrase gene in Acinetobacter baumannii and drug-resistance. Zhong Nan Da Xue Xue Bao Yi Xue Ban. 2008;33(10):952-957.

176. Martins N, Picão RC, Adams-Sapper S, Riley LW, Moreira BM. Association of class 1 and 2 integrons with multidrug-resistant Acinetobacter baumannii international clones and Acinetobacter nosocomialis isolates. Antimicrob Agents Chemother. 2015;59(1):698-701.

177. Kaliterna V, Kaliterna M, Hrenović J, Barišić Z, Tonkić M, Goic-Barisic I. Acinetobacter baumannii in Southern Croatia: clonal lineages, biofilm formation, and resistance patterns. Infect Dis. 2015;47(12):902-907.

178. Selasi GN, Nicholas A, Jeon H, et al. Differences in biofilm mass, expression of biofilm-associated genes, and resistance to desiccation between epidemic and sporadic clones of carbapenemresistant Acinetobacter baumannii sequence type 191. PloS One. 2016;11(9):e0162576.

179. Jang I-A, Kim J, Park W. Endogenous hydrogen peroxide increases biofilm formation by inducing exopolysaccharide production in $\mathrm{Aci}$ netobacter oleivorans DR1. Sci Rep. 2016;6.

180. Giannouli M, Antunes LC, Marchetti V, Triassi M, Visca P, Zarrilli R. Virulence-related traits of epidemic Acinetobacter baumannii strains belonging to the international clonal lineages I-III and to the emerging genotypes ST25 and ST78. BMC Infect Dis. 2013;13(1):282.

181. Wroblewska MM, Sawicka-Grzelak A, Marchel H, Luczak M, Sivan A. Biofilm production by clinical strains of Acinetobacter baumannii isolated frompatients hospitalized in two tertiary care hospitals. FEMS Immunol Med Microbiol. 2008;53(1):140-144.

182. de Campos PA, Royer S, da Fonseca Batistao DW, et al. Multidrug resistance related to biofilm formation in Acinetobacter baumannii and Klebsiella pneumoniae clinical strains from different pulsotypes. Curr Microbiol. 2016;72(5):617-627.

183. Park JY, Kim S, Kim S-M, Cha SH, Lim S-K, Kim J. Complete genome sequence of multidrug-resistant Acinetobacter baumannii strain 16562, which forms sturdy biofilm. J Bacteriol. 2011;193(22):6393-6394.
184. Hassan A, Naz A, Obaid A, et al. Pangenome and immuno-proteomics analysis of Acinetobacter baumannii strains revealed the core peptide vaccine targets. BMC Genomics. 2016;17(1):732.

185. Chopra S, Ramkissoon K, Anderson D. A systematic quantitative proteomic examination of multidrug resistance in Acinetobacter baumannii. J Proteomics. 2013;84:17-39.

186. Rumbo-Feal S, Gómez MJ, Gayoso C, et al. Whole transcriptome analysis of Acinetobacter baumannii assessed by RNA-sequencing reveals different mRNA expression profiles in biofilm compared to planktonic cells. PLoS One. 2013;8(8):e72968.

187. Lee S-Y, Yun SH, Lee YG, et al. Proteogenomic characterization of antimicrobial resistance in extensively drug-resistant Acinetobacter baumannii DU202. J Antimicrob Chemother. 2014;69(6): 1483-1491.

188. Murray GL, Tsyganov K, Kostoulias XP, et al. Global Gene expression profile of Acinetobacter baumannii during bacteremia. J Infect Dis. 2017;215(supp1_1):S52-S57.

189. Yeom J, Shin J-H, Yang J-Y, Kim J, Hwang G-S. 1H NMR-based metabolite profiling of planktonic and biofilm cells in Acinetobacter baumannii 1656-2. PloS One. 2013;8(3):e57730.

190. Hawkey PM, Warren RE, Livermore DM, et al. Treatment of infections caused by multidrug-resistant Gram-negative bacteria: report of the British Society for Antimicrobial Chemotherapy/healthcare Infection Society/british Infection Association Joint Working Party. J Antimicrob Chemother. 2018;73(suppl_3):iii2-iii78.

191. Sader HS, Castanheira M, Flamm RK, Mendes RE, Farrell DJ, Jones RN. Ceftazidime/avibactam tested against Gram-negative bacteria from intensive care unit (ICU) and non-ICU patients, including those with ventilatorassociated pneumonia. Int J Antimicrob Agents. 2015;46(1):53-59.

192. Li T, Sheng M, Gu T, Zhang Y, Yirepanjiang A, Li Y. In vitro assessment of cefoperazone-sulbactam based combination therapy for multidrugresistant Acinetobacter baumannii isolates in China. J Thorac Dis 2018;10(3):1370.

193. Lapuebla A, Abdallah M, Olafisoye O, et al. Activity of imipenem with relebactam against Gram-negative pathogens from New York City. Antimicrob Agents Chemother. 2015;59(8):5029-5031.

194. Sezgin FM, Coban AY, Gunaydin M. Investigation of biofilm formation in Acinetobacter baumannii isolates and their colistin susceptibilities in biofilm. Int J Antimicrob Agents. 2013;41(2):199.

195. Gaidhani SV, Raskar AV, Poddar S, et al. Time dependent enhanced resistance against antibiotics \& metal salts by planktonic \& biofilm form of Acinetobacter haemolyticus MMC 8 clinical isolate. Indian J Med Res. 2014;140(5):665.

196. Ozbek B, Mataraci E. In vitro effectiveness of colistin, tigecycline and levofloxacin alone and combined with clarithromycin and/or heparin as lock solutions against embedded Acinetobacter baumannii strains. J Antimicrob Chemother. 2012;68(4):827-830.

197. Ozbek B, Mataraci E. Comparative in vitro efficacies of colistinlevofloxacin, colistin-tigecycline and tigecycline-levofloxacin based catheter lock solutions on eradication of Acinetobacter baumannii biofilms. J Antimicrob Chemother. 2013; 2013;68(4):827-830.

198. Wang Y, Bao W, Guo N, et al. Antimicrobial activity of the imipenem/ rifampicin combination against clinical isolates of Acinetobacter baumannii grown in planktonic and biofilm cultures. World J Microbiol Biotechnol. 2014;30(12):3015-3025.

199. Song JY, Cheong HJ, Noh JY, Kim WJ. In vitro comparison of antibiofilm effects against carbapenem-resistant Acinetobacter baumannii: imipenem, colistin, tigecycline, rifampicin and combinations. Infect Chemother. 2015;47(1):27-32.

200. Wang Y-C, Kuo S-C, Yang Y-S, et al. Individual or combined effects of meropenem, imipenem, sulbactam, colistin, and tigecycline on biofilm-embedded Acinetobacter baumannii and biofilm architecture. Antimicrob Agents Chemother. 2016;60(8):4670-4676.

201. Kang YS, Park W. Contribution of quorum-sensing system to hexadecane degradation and biofilm formation in Acinetobacter sp. strain DR1. J Appl Microbiol. 2010;109(5):1650-1659. 
202. Chow JY, Yang Y, Tay SB, Chua KL, Yew WS. Disruption of biofilm formation by the human pathogen Acinetobacter baumannii using engineered quorum-quenching lactonases. Antimicrob Agents Chemother. 2014;58(3):1802-1805.

203. Zhang Y, Brackman G, Coenye T. Pitfalls associated with evaluating enzymatic quorum quenching activity: the case of MomL and its effect on Pseudomonas aeruginosa and Acinetobacter baumannii biofilms. PeerJ. 2017;5:e3251.

204. Perumal P, Wand M, Sutton J, Bock L. Evaluation of the effectiveness of hydrogen-peroxide-based disinfectants on biofilms formed by Gramnegative pathogens. J Hosp Infect. 2014;87(4):227-233.

205. Yele AB, Thawal ND, Sahu PK, Chopade BA. Novel lytic bacteriophage AB7-IBB1 of Acinetobacter baumannii: isolation, characterization and its effect on biofilm. Arch Virol. 2012;157(8):1441-1450.

206. Shi P, Gao Y, Lu Z, Yang L. Effect of antibacterial peptide LL-37 on the integrity of Acinetobacter baumannii biofilm. Nan Fang Yi Ke Da Xue Xue Bao. 2014;34(3):426-429.

207. Gordya N, Yakovlev A, Kruglikova A, et al. Natural antimicrobial peptide complexes in the fighting of antibiotic resistant biofilms: Calliphora vicina medicinal maggots. PloS One. 2017;12(3): e0173559.

208. Tutar U. Study of the effect of essential oil of Salvia glutinosa L. on microbial biofilm formation by clinical isolates of Acinetobacter baumannii. Paper presented at: AIP Conference Proceedings; Turkey; 2016.

209. Peng L, DeSousa J, Su Z, et al. Inhibition of Acinetobacter baumannii biofilm formation on a methacrylate polymer containing a 2-aminoimidazole subunit. Chem Commun. 2011;47(17):4896-4898.

210. Runci F, Bonchi C, Frangipani E, Visaggio D, Visca P. Acinetobacter baumannii biofilm formation in Human serum and disruption by gallium. Antimicrob Agents Chemother. 2017;61(1):e01563-01516.

211. Shakeri S, Kermanshahi RK, Moghaddam MM, Emtiazi G. Assessment of biofilm cell removal and killing and biocide efficacy using the microtiter plate test. Biofouling. 2007;23(2):79-86

212. Sambanthamoorthy K, Hickman M, Pattabiraman N, Palys T, Wagar EJ. Modulating Acinetobacter baumannii biofilm development with molecules containing 3, 4, 5-trimethoxy-N, $\mathrm{N}^{\prime}, \mathrm{N}^{\prime}$-trimethylbenzohydrazide moiety. Bioorg Med Chem Lett. 2015;25(10):2238-2242.

213. Tseng S-P, Hung W-C, Huang C-Y, et al. 5-Episinuleptolide decreases the expression of the extracellular matrix in early biofilm formation of multi-drug resistant Acinetobacter baumannii. Mar Drugs. 2016;14(8): 143 .

214. Fleeman R, Van Horn KS, Barber MM, et al. Characterizing the antimicrobial activity of N2, N4-disubstituted quinazoline-2, 4-diamines toward multidrug-resistant Acinetobacter baumannii. Antimicrob Agents Chemother. 2017;61(6):e00059-00017.
215. Chabane YN, Mlouka MB, Alexandre S, et al. Virstatin inhibits biofilm formation and motility of Acinetobacter baumannii. BMC Microbiol. 2014;14(1):62.

216. Ahmed NH, Baba K, Clay C, Lekalakala R, Hoosen AA. In vitro activity of tigecycline against clinical isolates of carbapenem resistant Acinetobacter baumannii complex in Pretoria, South Africa. BMC Res Notes. 2012;5(1):215.

217. Kateete DP, Nakanjako R, Namugenyi J, Erume J, Joloba ML, Najjuka CF. Carbapenem resistant Pseudomonas aeruginosa and Acinetobacter baumannii at Mulago Hospital in Kampala, Uganda (2007-2009). SpringerPlus. 2016;5(1):1308.

218. Doyle JS, Buising KL, Thursky KA, Worth LJ, Richards MJ. Epidemiology of infections acquired in intensive care units. Semin Respir Crit Care Med. 2011;32(2):115-138.

219. Al-Agamy MH, Khalaf NG, Tawfick MM, Shibl AM, El Kholy A. Molecular characterization of carbapenem-insensitive Acinetobacter baumannii in Egypt. Int J Infect Dis. 2014;22:49-54.

220. El-Sayed-Ahmed MA, Amin MA, Tawakol WM, Loucif L, Bakour $\mathrm{S}$, Rolain J-M. High prevalence of $b l a_{\mathrm{NDM}-1}$ carbapenemase-encoding gene and 16SrRNA armA methyltransferase among Acinetobacter baumannii clinical isolates, Egypt. Antimicrob Agents Chemother. 2015:59:3602-5.

221. Lopes BS, Al-Agamy MH, Ismail MA, et al. The transferability of bla OXA-23 gene in multidrug-resistant Acinetobacter baumannii isolates from Saudi Arabia and Egypt. Int J Med Microbiol. 2015;305(6):581-588.

222. Lowings M, Ehlers MM, Dreyer AW, Kock MM. High prevalence of oxacillinases in clinical multidrug-resistant Acinetobacter baumannii isolates from the Tshwane region, South Africa-an update. BMC Infect Dis. 2015;15(1):521.

223. World Health Organization. Global priority list of antibiotic-resistant bacteria to guide research, discovery, and development of new antibiotics. Available at: http://www.who.int/medicines/publications/ WHO-PPL-Short_Summary_25Feb-ET_NM_WHO.pdf. Accessed October 10, 2018.

224. Tacconelli E, Carrara E, Savoldi A et al. Discovery, research, and development of new antibiotics: the WHO priority list of antibioticresistant bacteria and tuberculosis. Lancet Infect Dis. 2018;18,318-327.

225. Hall CW, Mah TF. Molecular mechanisms of biofilm-based antibiotic resistance and tolerance in pathogenic bacteria. FEMS Microbiol Rev. 2017;41:276-301.

226. Mah TC, Toole, GA. Mechanisms of biofilm resistance to antimicrobial agents. Trends Microbiol. 2001;9:34-39.

227. Nickel JC, Ruseska I, Wright JB, Costerton JW. Tobramycin resistance of cells of Pseudomonas aeruginosa growing as biofilm on urinary catheter material. Antimicrob Agents Chemother. 1985;27:619-624.
Infection and Drug Resistance

\section{Publish your work in this journal}

Infection and Drug Resistance is an international, peer-reviewed openaccess journal that focuses on the optimal treatment of infection (bacterial, fungal and viral) and the development and institution of preventive strategies to minimize the development and spread of resistance. The journal is specifically concerned with the epidemiology of antibiotic
Dovepress

resistance and the mechanisms of resistance development and diffusion in both hospitals and the community. The manuscript management system is completely online and includes a very quick and fair peerreview system, which is all easy to use. Visit http://www.dovepress.com/ testimonials.php to read real quotes from published authors. 